\title{
Kaon generalized parton distributions and light-front wave functions in the Nambu-Jona-Lasinio model
}

\author{
Jin-Li Zhang ${ }^{\mathrm{a}} \mathbb{1}$, Jia-Lun Ping ${ }^{\mathrm{b}}$ (D) \\ Department of Physics, Nanjing Normal University, Nanjing 210023, China
}

Received: 25 June 2021 / Accepted: 28 August 2021 / Published online: 15 September 2021

(C) The Author(s) 2021

\begin{abstract}
Kaon generalized parton distributions (GPDs) and the leading Fock state light-front wave functions are investigated in the framework of Nambu-Jona-Lasinio model with proper time regularization. In addition, we compared the form factors, parton distribution functions, and generalized form factors obtained from them, respectively. The first Mellin moments of GPDs result in the form factors of local currents. The second Mellin moments of vector GPDs are related to gravitational form factors, the quark mass distribution $\theta_{2}$ and the quark pressure distribution $\theta_{1}$. When taking a Fourier transform of GPDs in impact parameter space, we can get the mean-squared impact parameter for the quarks of the kaon: $\left\langle\boldsymbol{b}_{\perp}^{2}\right\rangle_{K}^{u}=0.149 \mathrm{fm}^{2},\left\langle\boldsymbol{b}_{\perp}^{2}\right\rangle_{K}^{s}=0.088 \mathrm{fm}^{2}$. This means that the kaon $s$ quark is nearer to the center of transverse momentum than the $u$ quark. We also give the light-cone energy radius for the quarks of the kaon from the mass distribution $\theta_{2}: r_{E, L C}^{u, K}=0.187 \mathrm{fm}, r_{E, L C}^{s, K}=0.167 \mathrm{fm}$, and the light-cone charge radius from quark form factors of the kaon: $r_{c, L C}^{u, K}=0.390 \mathrm{fm}, r_{c, L C}^{s, K}=0.296 \mathrm{fm}$, which means that the $s$ quark has a smaller extent than the $u$ quark. The light-front transverse-spin distributions $\rho_{u}^{1}\left(\boldsymbol{b}_{\perp}, \boldsymbol{s}_{\perp}\right)$ and $\rho_{u}^{2}\left(\boldsymbol{b}_{\perp}, \boldsymbol{s}_{\perp}\right)$ show distortions, the average shift are $\left\langle b_{\perp}^{y}\right\rangle_{1}^{u}=0.116 \mathrm{fm}$ and $\left\langle b_{\perp}^{y}\right\rangle_{2}^{u}=0.083 \mathrm{fm}$. On the kinematic domain associated with the valence-quark dominance, the unpolarized Wigner distribution from light-front wave functions is sharply peaked. It extends as the transverse position variable increases in magnitude and has a domain of negative support. Through the comparison of distributions from the two methods, we find that they give the same multi-dimensional mapping of the kaon in the Nambu-Jona-Lasinio model.
\end{abstract}

\footnotetext{
a e-mail: jlzhang@njnu.edu.cn (corresponding author)

b e-mail: jlping@njnu.edu.cn
}

\section{Introduction}

Generalized parton distributions (GPDs) [1-9] were proposed in the 1990s to describe the multi-dimensional pictures of hadrons. GPDs include a mass of inaccessible information about the partonic structure of hadrons; therefore, GPDs have been under thorough theoretical and experimental studies ever since. Experimentally, GPDs offer a solid formal basis to link information from multifarious inclusive, semiinclusive and exclusive processes in an efficient, explicit way. Hard exclusive processes, such as deeply virtual Compton scattering (DVCS) [2,10-12] and deeply virtual meson production (DVMP) [13,14], in the asymptotic regime, can be expressed in terms of GPDs. The publications of new experimental data $[15,16]$ have increased the interest in the study of GPDs; the upgraded Jefferson Laboratory facility 12 can offer very precise experimental data in the near future, this will challenge the present understanding of GPDs and furthermore the present models of GPDs. Theoretically, the proper description of GPDs plays an important role in obtaining insight into the internal structure of hadrons. Comparing with the available experimental data, using the phenomenological models, the framework of GPDs are well built now, providing a three-dimensional picture of the hadrons $[4,6]$. GPDs provide more than the ordinary parton distribution functions (PDFs) [17-26] and form factors (FFs) [27-32] in understanding the internal structure of hadrons. Besides the longitudinal-momentum fraction $x$ and the momentum transfer $q^{2}$, GPDs also depend on the skewness parameter $\xi$. GPDs contain information of both the spatial and the momentum quark distributions in a hadron. GPDs can also provide information that can define the internal structure and dynamics of the hadron: the mechanical properties of the angular momentum [2,33], pressure, and shear forces [34,35]. Therefore, GPDs can be treated as a useful tool to get the transverse spatial distribution of partons. 
On the other hand, light-front wave functions (LFWFs) [36-43] of an interacting quantum system provide a connection between hadrons and their fundamental quark and gluon degrees of freedom in quantum chromodynamics (QCD). The features that appear purely through the infinitely-manybody nature of relativistic quantum field theory can be translated into images whose explanation appears to be more straightforward. LFWFs can describe the hard exclusive processes in terms of a nonperturbative hadron, thus can calculate a wide range of physical hadronic observables, such as, FFs, PDFs, GPDs, parton distribution amplitudes (PDAs) [44-49], and transverse momentum dependent parton distribution (TMDs) [50-62]. So in addition to calculating these distributions directly from the Feynman diagrams, another fruitful idea used in model building is to construct these distributions from LFWFs.

LFWFs and GPDs are hard to calculate from QCD directly, the phenomenological parametrizations are successful, but still need to be improved. Thus, several modeling frameworks have been used in the last few decades, each one having its advantages. For instance, we have the NambuJona-Lasinio (NJL) model [63-68], the constituent quark model [69-71], the light-front holographic model [72-74], the light-front quark-diquark model [75-77], the AdS/QCD model [78-80], the chiral quark model $[81,82]$ and the Dyson-Schwinger equations (DSEs) $[7,8,83]$. The NJL model has an effective Lagrangian of relativistic fermions interacting through local fermion-fermion couplings, and especially, it preserves one of the most important fundamental symmetries of QCD, namely, chiral symmetry. NJL results are to be identified as qualitatively sound initial conditions for use in exploring the domain of physical possibilities in areas where more realistic frameworks are not yet able to provide insights or predictions, so that is widely used in many fields [63,84-87]. In this paper, we will use the NJL model to derive LFWFs and GPDs.

This paper is constructed as follows: In Sect. 2, we give a brief introduction of the NJL model, then the definition and calculation of the kaon GPDs, in addition, the fundamental properties of kaon GPDs will be examined in this section. In Sect. 3, kaon LFWFs in the NJL model will be discussed, the relationship of LFWFs with the PDFs, FFs, PDAs and GPDs will be given. Finally, we give a brief summary and outlook in Sect. 4.

\section{Generalized parton distributions}

Different from the diagonal parton distributions, GPDs are determined by matrix elements of quark and gluon lightcone operators for distinct initial and final states of hadron. GPDs include richer information than the ordinary parton distributions, thus they cannot be interpreted as probabilities directly. We will express GPDs in a symmetric manner as in Refs. [4,6], with a skewing parameter $\xi$. In this section, we will calculate kaon vector and tensor GPDs in the NJL model using proper time regularization (PTR).

\subsection{Nambu-Jona-Lasinio model}

The SU(3) flavor NJL Lagrangian in the $\bar{q} q$ interaction channel we take in the form [88]

$\mathcal{L}=\bar{\psi}\left(i \gamma^{\mu} \partial_{\mu}-\hat{m}\right) \psi+G_{\pi}\left[\left(\bar{\psi} \lambda_{a} \psi\right)^{2}-\left(\bar{\psi} \gamma_{5} \lambda_{a} \psi\right)^{2}\right]$,

where the quark field has the flavor components $\psi^{T}=$ $(u, d, s), \lambda_{a}, a=0, \ldots, 8$ are the eight Gell-Mann matrices in flavor space where $\lambda_{0}=\sqrt{2 / 3} \nVdash . \hat{m}=\operatorname{diag}\left(m_{u}, m_{d}, m_{s}\right)$ is the current quark mass matrix. $G_{\pi}$ is an effective coupling strength of the scalar $\left(\bar{q} \lambda_{a} q\right)$ and pseudoscalar $\left(\bar{q} \gamma_{5} \lambda_{a} q\right)$ interaction channel.

The dressed-quark propagator in the NJL model is obtained by solving the gap equation in Fig. 1 ,

$S_{q}(k)=\frac{1}{\not k-M_{q}+i \varepsilon}$,

where $M_{q}=\left(M_{u}, M_{d}, M_{s}\right)$ is the dressed-quark mass. The interaction kernel of the gap equation in Fig. 1 is local so we get a constant dressed-quark mass $M_{q}$, which satisfies

$M_{q}=m_{q}+12 i G_{\pi} \int \frac{\mathrm{d}^{4} l}{(2 \pi)^{4}} \operatorname{Tr}_{D}\left[S_{q}(l)\right]$,

where the trace is over Dirac indices. From the above equation we can see that in contrast with the SU(2) flavor case, flavor mixing is absent in the SU(3) flavor case [85,89]. Moreover, dynamical chiral symmetry breaking can take place only when the coupling constant $G_{\pi}>G_{\text {critical }}$, which gives a nontrivial solution $M_{q}>0$.

In the NJL model, the description of mesons as $\bar{q} q$ bound states is obtained through the Bethe-Salpeter equation (BSE). The solution of the BSE in each meson channel is given by a two-body $t$-matrix that depends on the nature of the interaction channel, where the reduced $t$-matrices for the kaon meson reads

$\tau_{K}(q)=\frac{-2 i G_{\pi}}{1+2 G_{\pi} \Pi_{P P}\left(q^{2}\right)}$,

where the bubble diagram $\Pi_{P P}\left(q^{2}\right)$ is defined as

$\Pi_{P P}\left(q^{2}\right) \delta_{i j}=3 i \int \frac{\mathrm{d}^{4} k}{(2 \pi)^{4}} \operatorname{Tr}\left[\gamma^{5} \lambda_{i} S_{u}(k) \gamma^{5} \lambda_{j} S_{S}(k+q)\right]$, 


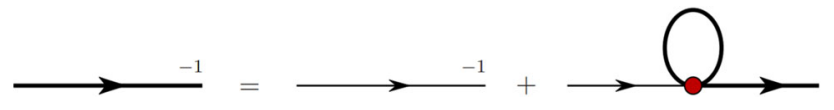

Fig. 1 The NJL gap equation in the Hartree-Fock approximation, where the thin line represents the elementary quark propagator, the shaded circle represents the $\bar{q} q$ interaction kernel. Higher-order terms, attributed to meson loops for example, are not included in the gap equation kernel

where the traces are over Dirac and isospin indices, for the kaon $i, j=4,5,6,7$. The mass of the kaon is given by the pole in the reduced $t$-matrix,

$1+2 G_{\pi} \Pi_{P P}\left(q^{2}=m_{K}^{2}\right)=0$.

Expanding the full $t$-matrix about the pole gives the homogeneous Bethe-Salpeter vertex for the kaon,

$\Gamma_{K}^{i}=\sqrt{Z_{K}} \gamma_{5} \lambda_{i}$

the normalization factor is given by

$Z_{K}^{-1}=-\left.\frac{\partial}{\partial q^{2}} \Pi_{P P}\left(q^{2}\right)\right|_{q^{2}=m_{K}^{2}}$.

This residue can be explained as the square of effective meson-quark-quark coupling constant. Homogeneous Bethe-Salpeter vertex functions are an essential ingredient in, for example, triangle diagrams that determine the meson form factors.

The NJL model is a non-renormalizable model, so a regularization scheme is needed. We will use the PTR scheme [90-92],

$$
\begin{aligned}
\frac{1}{X^{n}} & =\frac{1}{(n-1) !} \int_{0}^{\infty} \mathrm{d} \tau \tau^{n-1} e^{-\tau X} \\
& \rightarrow \frac{1}{(n-1) !} \int_{1 / \Lambda_{\mathrm{UV}}^{2}}^{1 / \Lambda_{\mathrm{IR}}^{2}} \mathrm{~d} \tau \tau^{n-1} e^{-\tau X},
\end{aligned}
$$

where $X$ represents a product of propagators that have been connected using Feynman parametrization. $\Lambda_{\mathrm{UV}}$ is the ultraviolet cutoff. The NJL model does not contain confinement, the infrared cutoff is used to mimic it, therefore it should be of the order $\Lambda_{\mathrm{QCD}}$ and we choose $\Lambda_{\mathrm{IR}}=0.240 \mathrm{GeV}$. For the light-quark dressed masses we choose $M_{u}=M_{d}=0.4$ $\mathrm{GeV}$; the ultraviolet cutoff $\Lambda_{\mathrm{UV}}$ and the coupling $G_{\pi}$ are constrained by the empirical values of pion decay constant and pion mass. The kaon is regarded as a relativistic bound state of a dressed quark and a dressed antiquark whose properties are determined by solving the $\bar{q} q$ Bethe-Salpeter equation in the pseudoscalar channel. In Table 1, we give the parameters used in this paper.

In the following, we will use the $\mathcal{C}$ functions and formulas as explained in the appendix.
Table 1 Parameter set used in our work. The dressed-quark mass and regularization parameters are in units of $\mathrm{GeV}$, while coupling constant are in units of $\mathrm{GeV}^{-2}$

\begin{tabular}{lllllll}
\hline$\Lambda_{\mathrm{IR}}$ & $\Lambda_{\mathrm{UV}}$ & $M_{u}$ & $M_{s}$ & $G_{\pi}$ & $m_{K}$ & $Z_{K}$ \\
\hline 0.240 & 0.645 & 0.40 & 0.59 & 19.0 & 0.47 & 20.47 \\
\hline
\end{tabular}

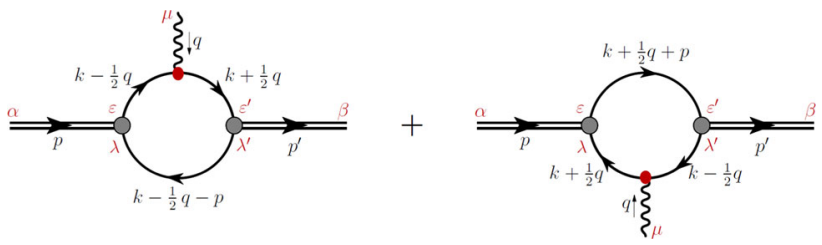

Fig. 2 Kaon GPDs diagrams

\subsection{The definition and calculation of the kaon GPDs}

In the NJL model, kaon GPDs are given by Fig. 2, where $p$ is the initial and $p^{\prime}$ is the final kaon momentum, the kinematics and related quantities of this process are defined as

$p^{2}=p^{\prime 2}=m_{K}^{2}, \quad t=q^{2}=\left(p^{\prime}-p\right)^{2}=-Q^{2}$,

$P=\frac{p+p^{\prime}}{2}, \quad \xi=\frac{p^{+}-p^{+}}{p^{+}+p^{\prime+}}, \quad n^{2}=0$,

where $\xi$ is the so called skewness parameter, $n$ is the lightcone four-vector defined as $n=(1,0,0,-1)$, in the lightcone coordinate

$v^{ \pm}=\left(v^{0} \pm v^{3}\right), \quad \mathbf{v}=\left(v^{1}, v^{2}\right)$,

for any four-vector, $v^{+}$in the light-cone coordinate can be defined as

$v^{+}=v \cdot n$

The vector and tensor quark GPDs of the kaon are defined as

$$
\begin{aligned}
& H^{q}(x, \xi, t)=\frac{1}{2} \int \frac{\mathrm{d} z^{-}}{2 \pi} e^{\frac{i}{2} x\left(p^{+}+p^{++}\right) z^{-}} \\
& \quad \times\left.\left\langle p^{\prime}\left|\bar{q}\left(-\frac{1}{2} z\right) \gamma^{+} q\left(\frac{1}{2} z\right)\right| p\right\rangle\right|_{z^{+}=0, z=0,} \\
& \frac{P^{+} q^{j}-P^{j} q^{+}}{P^{+} m_{K}} E^{q}(x, \xi, t)=\frac{1}{2} \int \frac{\mathrm{d} z^{-}}{2 \pi} e^{\frac{i}{2} x\left(p^{+}+p^{++}\right) z^{-}} \\
& \quad \times\left.\left\langle p^{\prime}\left|\bar{q}\left(-\frac{1}{2} z\right) i \sigma^{+j} q\left(\frac{1}{2} z\right)\right| p\right\rangle\right|_{z^{+}=0, z=0},
\end{aligned}
$$

where $x$ is the longitudinal-momentum fraction. $H^{q}(x, \xi, t)$ is the no spin flip or vector GPD, $E^{q}(x, \xi, t)$ is the spin flip or tensor GPD. 
In Fig. 2, we give the Feynman diagrams of the kaon GPDs, where the operators in the diagram have the form

$$
\begin{aligned}
& \bullet_{1}=\gamma^{+} \delta\left(x-\frac{k^{+}}{P^{+}}\right), \\
& \bullet 2=i \sigma^{+j} \delta\left(x-\frac{k^{+}}{P^{+}}\right),
\end{aligned}
$$

where the first is for the vector GPD and the second for the tensor GPD.

In the NJL model, the $u$ quark vector and tensor GPDs of $\mathrm{K}^{+}$meson are defined as

$$
\begin{aligned}
& H^{u}(x, \xi, t)=2 i N_{c} Z_{K} \int \frac{\mathrm{d}^{4} k}{(2 \pi)^{4}} \delta_{n}^{x}(k) \\
& \times \operatorname{Tr}\left[\gamma_{5} S_{u}\left(k_{+}\right) \gamma^{+} S_{u}\left(k_{-}\right) \gamma_{5} S_{s}\left(k_{P}\right)\right], \\
& \frac{P^{+} q^{j}-P^{j} q^{+}}{P^{+} m_{K}} E^{u}(x, \xi, t) \\
& =2 i N_{c} Z_{K} \int \frac{\mathrm{d}^{4} k}{(2 \pi)^{4}} \delta_{n}^{x}(k) \\
& \quad \times \operatorname{Tr}\left[\gamma_{5} S_{u}\left(k_{+}\right) i \sigma^{+j} S_{u}\left(k_{-}\right) \gamma_{5} S_{S}\left(k_{P}\right)\right],
\end{aligned}
$$

where $\delta_{n}^{x}(k)=\delta\left(x P^{+}-k^{+}\right) k_{+}=k+\frac{q}{2}, k_{-}=k-\frac{q}{2}$, $k_{P}=k-P$.

Here we use the notations

$$
\begin{aligned}
& \mathcal{D}_{k_{+}}^{u}=\left(k+\frac{q}{2}\right)^{2}-M_{u}^{2}, \\
& \mathcal{D}_{k_{-}}^{u}=\left(k-\frac{q}{2}\right)^{2}-M_{u}^{2}, \\
& \mathcal{D}_{k_{P}}^{s}=\left(k-p-\frac{q}{2}\right)^{2}-M_{s}^{2},
\end{aligned}
$$

we can get the following reduced formulas:

$$
\begin{aligned}
p \cdot q & =-\frac{q^{2}}{2} \\
k \cdot q & =\frac{1}{2}\left(\mathcal{D}_{k_{+}}^{u}-\mathcal{D}_{k_{-}}^{u}\right) \\
k \cdot p & =-\frac{1}{2}\left(\mathcal{D}_{k_{P}}^{s}-\mathcal{D}_{k_{-}}^{u}-m_{K}^{2}-M_{u}^{2}+M_{s}^{2}+\frac{q^{2}}{2}\right) \\
k^{2} & =\frac{1}{2}\left(\mathcal{D}_{k_{+}}^{u}+\mathcal{D}_{k_{-}}^{u}\right)+M_{u}^{2}-\frac{q^{2}}{4}
\end{aligned}
$$

after some calculation we arrive at

$$
\begin{aligned}
& H^{u}(x, \xi, t) \\
& \quad=\frac{N_{c} Z_{K}}{8 \pi^{2}}\left[\theta_{\bar{\xi} 1} \overline{\mathcal{C}}_{1}\left(\sigma_{3}\right)+\theta_{\xi 1} \overline{\mathcal{C}}_{1}\left(\sigma_{4}\right)+\frac{\theta_{\bar{\xi} \xi}}{\xi} x \overline{\mathcal{C}}_{1}\left(\sigma_{5}\right)\right]
\end{aligned}
$$

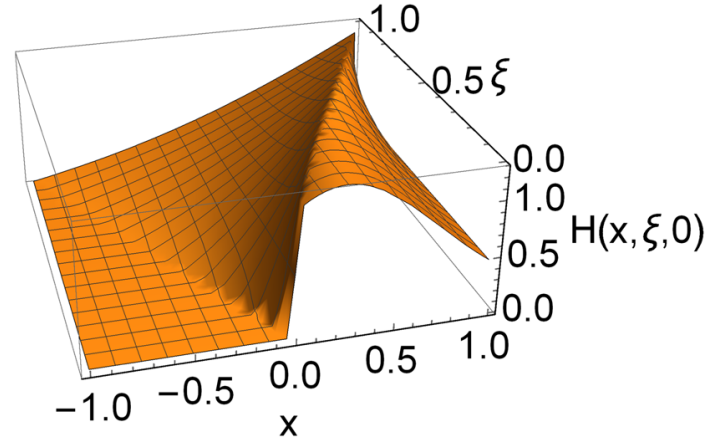

Fig. 3 Kaon vector GPD: left panel $-H^{u}(x, \xi, 0)$ in Eq. (21), because of the symmetry property in Eq. (27a), we only plot $\xi>0$ part

$$
\begin{aligned}
& \quad+\frac{N_{c} Z_{K}}{8 \pi^{2}} \int_{0}^{1} \mathrm{~d} \alpha \frac{\theta_{\alpha \xi}}{\xi} \frac{1}{\sigma_{6}} \overline{\mathcal{C}}_{2}\left(\sigma_{6}\right) \\
& \quad \times\left((1-x) t+2 x\left(m_{K}^{2}-\left(M_{u}-M_{s}\right)^{2}\right)\right), \\
& E^{u}(x, \xi, t) \\
& =\frac{N_{c} Z_{K}}{4 \pi^{2}} \int_{0}^{1} \mathrm{~d} \alpha \frac{\theta_{\alpha \xi}}{\xi} m_{K}\left(\left(M_{s}-M_{u}\right) \alpha+M_{u}\right) \frac{1}{\sigma_{6}} \overline{\mathcal{C}}_{2}\left(\sigma_{6}\right),
\end{aligned}
$$

and

$\theta_{\bar{\xi} 1}=x \in[-\xi, 1]$,

$\theta_{\xi 1}=x \in[\xi, 1]$,

$\theta_{\bar{\xi} \xi}=x \in[-\xi, \xi]$,

$\theta_{\alpha \xi}=x \in[\alpha(\xi+1)-\xi, \alpha(1-\xi)+\xi] \cap x \in[-1,1]$,

where $\theta$ is the step function, and $\theta$ function is 1 in the corresponding region, otherwise it is zero. These results are in the region $\xi>0$, under $\xi \rightarrow-\xi: \theta_{\bar{\xi} 1} \leftrightarrow \theta_{\xi 1}$; and $\theta_{\bar{\xi} \xi} / \xi$, $\theta_{\alpha \xi} / \xi$ are invariant. The diagrams of vector and tensor GPDs are plotted in Figs. 3, 4, 5 and 6. From the diagrams we can see that $H^{u}(x, \xi, 0)=0$ when $x<-\xi$, the vector GPD is continuous but not differentiable at $x= \pm \xi$ except $\xi=0$, the vector GPD is not continuous at $\xi=0$, the tensor GPD is similar. The properties of GPDs, for example, in the forward limit it should reduce to PDF, we will check these properties separately in this section.

\subsection{The properties of the kaon GPDs}

\subsubsection{Forward limit}

In the case that the initial and final kaon have the same momentum $p=p^{\prime}$, which means $\xi=0, t=0$, the vector GPD reduces to the kaon PDF, 


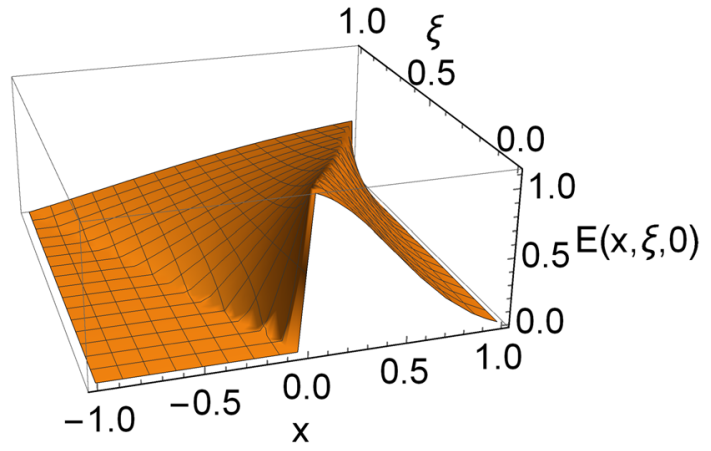

Fig. 4 Kaon tensor GPD $E^{u}(x, \xi, 0)$, because of the symmetry property in Eq. (27b), we only plot $\xi>0$ part

$$
\begin{aligned}
& u_{K}(x) \\
= & \frac{3 Z_{K}}{4 \pi^{2}} \overline{\mathcal{C}}_{1}\left(\sigma_{1}\right) \\
+ & \frac{3 Z_{K}}{2 \pi^{2}} x(1-x)\left(m_{K}^{2}-\left(M_{u}-M_{s}\right)^{2}\right) \frac{1}{\sigma_{1}} \overline{\mathcal{C}}_{2}\left(\sigma_{1}\right),
\end{aligned}
$$

which satisfies

$$
\int_{0}^{1} u_{K}(x) \mathrm{d} x=1 .
$$

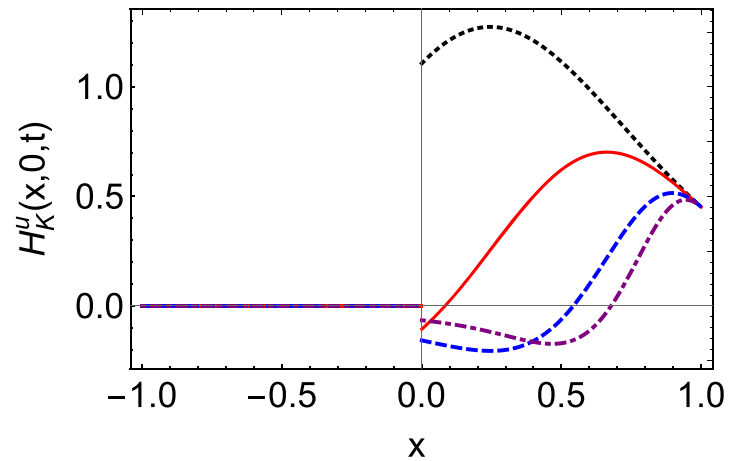

In Fig. 3, when $\xi=0, H^{u}(x, 0,0)$ is 0 when $x \in[-1,0]$, in the region $x \in[0,1]$, this $u$ quark PDF of the kaon is different from the pion PDF in the NJL model [9].

\subsubsection{Symmetry properties}

In general, GPDs are neither even nor odd of $x$, the combinations,

$H^{I=0}(x, \xi, t)=H^{u}(x, \xi, t)-H^{u}(-x, \xi, t)$,

$H^{I=1}(x, \xi, t)=H^{u}(x, \xi, t)+H^{u}(-x, \xi, t)$,

are useful, the first equation corresponds to charge conjugation $C=+1$ in the T-channel. The second equation corresponds to $C=-1$ in the T-channel, the tensor GPD $E^{u}(x, \xi, t)$ is analogous.

Our results preserve the time reversal invariance property of GPDs,

$\begin{aligned} H^{u}(x, \xi, t) & =H^{u}(x,-\xi, t), \\ E^{u}(x, \xi, t) & =E^{u}(x,-\xi, t),\end{aligned}$

we can establish this straightforwardly using the results following Eqs. (23) and (A2).

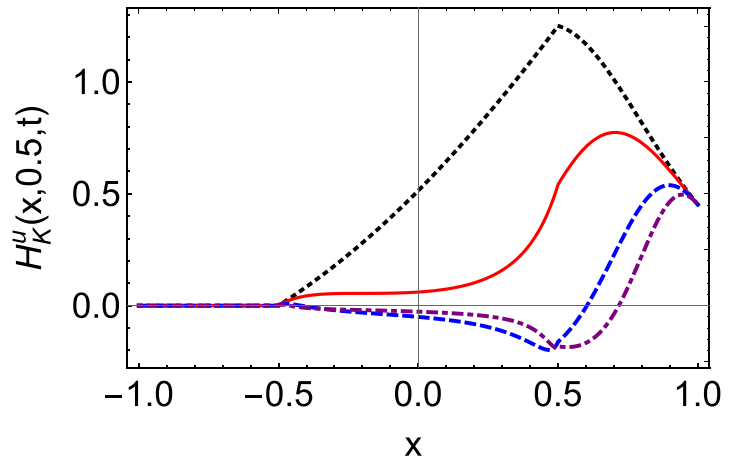

Fig. 5 Kaon $u$ quark vector GPDs (left panel: $\xi=0$, right panel: $\xi=0.5$ ) with different $t$ : black dotted line $-t=0 \mathrm{GeV}^{2}$, red line $-t=-1$ $\mathrm{GeV}^{2}$, blue dashed line $-t=-5 \mathrm{GeV}^{2}$, purple dotdashed line $-t=-10 \mathrm{GeV}^{2}$
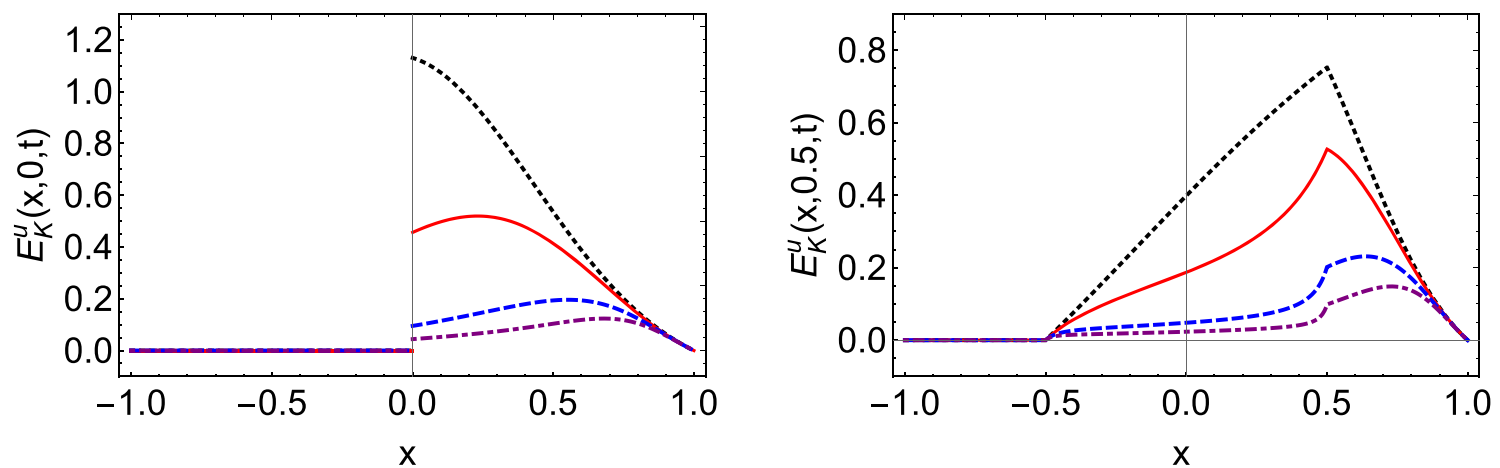

Fig. 6 Kaon $u$ quark tensor GPDs (left panel: $\xi=0$, right panel: $\xi=0.5$ ) with different $t$ : black dotted line $-t=0 \mathrm{GeV}^{2}$, red line $-t=-1$ $\mathrm{GeV}^{2}$, blue dashed line $-t=-5 \mathrm{GeV}^{2}$, purple dotdashed line $-t=-10 \mathrm{GeV}^{2}$ 


\subsubsection{Polynomiality condition}

When integrating $x^{n}$ moments of GPDs, one can get the polynomiality condition

$$
\begin{aligned}
& \int_{-1}^{1} x^{n} \mathrm{~d} x H^{q}(x, \xi, t)=\sum_{i=0}^{[(n+1) / 2]} \xi^{2 i} A_{n+1,2 i}^{q}(t), \\
& \int_{-1}^{1} x^{n} \mathrm{~d} x E^{q}(x, \xi, t)=\sum_{i=0}^{[(n+1) / 2]} \xi^{2 i} B_{n+1,2 i}^{q}(t),
\end{aligned}
$$

which is the remarkable polynomiality property of GPDs. When $n=0$, we get the FFs

$$
\begin{aligned}
& \int_{-1}^{1} \mathrm{~d} x H^{u}(x, \xi, t)=A_{1,0}^{u}(t)=F_{K}^{u}\left(Q^{2}\right), \\
& \int_{-1}^{1} \mathrm{~d} x E^{u}(x, \xi, t)=B_{1,0}^{u}(t)=F_{T}^{u}\left(Q^{2}\right),
\end{aligned}
$$

where $F_{K}^{u}\left(Q^{2}\right)$ and $F_{T}^{u}\left(Q^{2}\right)$ are the $u$ quark vector FF and tensor FF. $F_{K}^{u}\left(Q^{2}\right)$ is the electromagnetic form factor of the kaon, $F_{T}^{u}(0)$ is the tensor anomalous magnetic moment for $n=0$ [93]. We have

$$
\begin{aligned}
F_{K}^{u}\left(Q^{2}\right)= & \frac{N_{c} Z_{K}}{4 \pi^{2}} \int_{0}^{1} \mathrm{~d} x \overline{\mathcal{C}}_{1}\left(\sigma_{1}\right) \\
& +\frac{N_{c} Z_{K}}{4 \pi^{2}} \int_{0}^{1} \mathrm{~d} x \int_{0}^{1-x} \mathrm{~d} y \frac{1}{\sigma_{7}} \overline{\mathcal{C}}_{2}\left(\sigma_{7}\right) \\
& \times\left(2(1-x-y)\left(m_{K}^{2}-\left(M_{u}^{2}-M_{s}\right)^{2}\right)-(x+y) Q^{2}\right) \\
E_{T}^{u}\left(Q^{2}\right)= & \frac{N_{c} Z_{K}}{2 \pi^{2}} \int_{0}^{1} \mathrm{~d} x \int_{0}^{1-x} \mathrm{~d} y \\
& \times m_{K}\left(\left(M_{u}-M_{s}\right)(x+y)+M_{s}\right) \frac{1}{\sigma_{7}} \overline{\mathcal{C}}_{2}\left(\sigma_{7}\right)
\end{aligned}
$$

When $n=1$, the GPDs should follow the sum rule:

$$
\begin{aligned}
\int_{-1}^{1} x \mathrm{~d} x H^{u}(x, \xi, t) & =A_{2,0}^{u}(t)+\xi^{2} A_{2,2}^{u}(t) \\
& =\theta_{2}^{u}(t)-\xi^{2} \theta_{1}^{u}(t), \\
\int_{-1}^{1} x \mathrm{~d} x E^{u}(x, \xi, t) & =B_{2,0}^{u}(t)+\xi^{2} B_{2,2}^{u}(t),
\end{aligned}
$$

where $\theta_{2}^{u}$ relates to the $u$ quark mass distribution within the kaon and $\theta_{1}^{u}$ is associated with the $u$ quark pressure distribution. The polynomial only has the $\xi^{0}$ and $\xi^{2}$ term, this satisfies Eq. (28). The $n=2$ case has also been checked, it also satisfies the polynomiality condition. The generalized FFs for $n=1$ are

$$
A_{2,0}^{u}\left(Q^{2}\right)=\frac{N_{c} Z_{K}}{4 \pi^{2}} \int_{0}^{1} \mathrm{~d} x x \overline{\mathcal{C}}_{1}\left(\sigma_{1}\right)
$$

$$
\begin{aligned}
+ & \frac{N_{c} Z_{K}}{4 \pi^{2}} \int_{0}^{1} \mathrm{~d} x \int_{0}^{1-x} \mathrm{~d} y(1-x-y) \frac{1}{\sigma_{7}} \overline{\mathcal{C}}_{2}\left(\sigma_{7}\right) \\
& \times\left(2\left(m_{K}^{2}-\left(M_{s}-M_{u}\right)^{2}\right)(1-x-y)-Q^{2}(x+y)\right), \\
A_{2,2}^{u}\left(Q^{2}\right)= & -\frac{N_{c} Z_{K}}{2 \pi^{2}} \int_{0}^{1} \mathrm{~d} x x(1-2 x) \overline{\mathcal{C}}_{1}\left(\sigma_{2}\right) \\
& -\frac{N_{c} Z_{K}}{4 \pi^{2}} \int_{0}^{1} \mathrm{~d} x(1-x) \overline{\mathcal{C}}_{1}\left(\sigma_{1}\right) \\
& +\frac{N_{c} Z_{K}}{4 \pi^{2}} \int_{0}^{1} \mathrm{~d} x \\
& \times \frac{(1-x)\left(2\left(m_{K}^{2}-\left(M_{s}^{2}-M_{u}^{2}\right)\right)+Q^{2}\right) \overline{\mathcal{C}}_{1}\left(\sigma_{1}\right)}{} \\
& -\frac{N_{c} Z_{K}}{4 \pi^{2}} \int_{0}^{1} \mathrm{~d} x \int_{0}^{1-x} \mathrm{~d} y \\
& \times \frac{\left(2\left(m_{K}^{2}-\left(M_{s}-M_{u}\right)^{2}\right)+Q^{2}\right)}{Q_{1}}\left(\sigma_{7}\right) \\
B^{2} & \frac{N_{c} Z_{K}}{2 \pi^{2}} \int_{0}^{1} \mathrm{~d} x \int_{0}^{1-x} \mathrm{~d} y \frac{1}{\sigma_{7}} \overline{\mathcal{C}}_{2}\left(\sigma_{7}\right) \\
& \times m_{K}\left((1-x-y)^{2} M_{s}+(1-x-y)(x+y) M_{u}\right)
\end{aligned}
$$

for the $u$ quark tensor GPD $E^{u}(x, \xi, t)$ of the kaon in the NJL model, $B_{2,2}^{u}\left(Q^{2}\right)=0$. We have plotted these FFs in Fig. 7; from the diagram we can see that $\theta_{1}^{u}$ is harder than $\theta_{2}^{u}$ and both of them are harder than the vector FF, the tensor anomalous magnetic moment $B_{1,0}^{u}$ is softer than $B_{2,0}^{u}$.

A light-cone energy radius can be defined in relation to the generalized FF $A_{2,0}\left(Q^{2}\right)$ defined as [94]

$$
\begin{aligned}
& \left\langle r_{E, L C}^{2}\right\rangle=-\left.4 \frac{\partial A_{2,0}\left(Q^{2}\right)}{\partial Q^{2}}\right|_{Q^{2}=0}, \\
& \left\langle r_{c, L C}^{2}\right\rangle=-\left.4 \frac{\partial F_{K}\left(Q^{2}\right)}{\partial Q^{2}}\right|_{Q^{2}=0},
\end{aligned}
$$

the second equation is analogous to the light-cone charge radius, which can be compared with the light-cone energy radius. We get $r_{E, L C}^{u, K}=0.187 \mathrm{fm}, r_{E, L C}^{s, K}=0.167 \mathrm{fm}$, $r_{c, L C}^{u, K}=0.390 \mathrm{fm}$ and $r_{c, L C}^{s, K}=0.296 \mathrm{fm}$. In these two cases, the $s$ quark has a smaller extent than the $u$ quark. Comparing with the results of pion $r_{E, L C}^{u, \pi}=0.188 \mathrm{fm}, r_{c, L C}^{u, \pi}=0.374$ fm, then $r_{E, L C}^{u, \pi} / r_{c, L C}^{u, \pi}=0.502$. In Ref. [95], their ratio is 0.56 from the leading Fock state LFWF of pion using DSEs, and our former results demonstrate that the pion $A_{2,0}$ from the leading Fock state LFWF is the same as our direct calculation from the vector GPD [9]. Therefore, the values are the same, our ratio is smaller than their results. The kaon $A_{2,0}$ is similar in the NJL model, $r_{E, L C}^{u, K} / r_{c, L C}^{u, K}=0.479$, which is smaller than the pion case, while $r_{E, L C}^{s, K} / r_{c, L C}^{s, K}=0.564$ is bigger. These radii will be impacted by higher Fock states. 


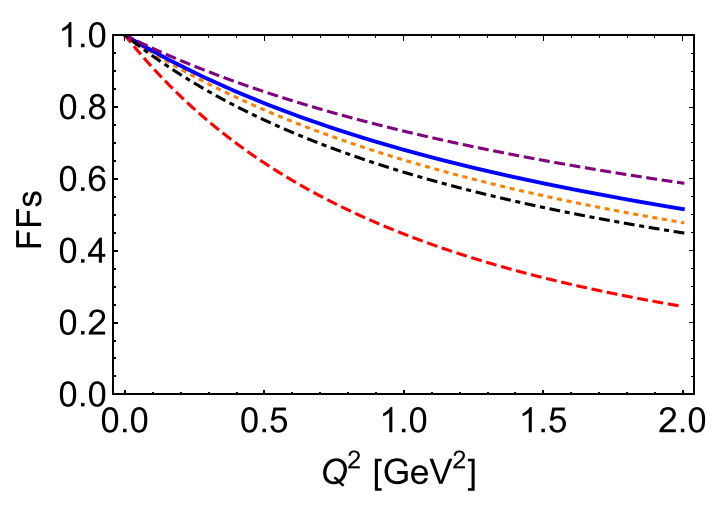

Fig. 7 Form factors normalized to their own charge: $F_{K}^{u}$-dashed red curve, $B_{1,0}^{u}$ - dot-dashed black curve, $A_{2,0}^{u}$ - dotted orange curve, $A_{2,2}^{u}$ - solid blue curve, $B_{2,0}^{u}$ - dashed purple curve

\subsubsection{Impact parameter dependent PDFs}

In order to localize the hadron in the transverse direction, we introduce

$\left|p^{+}, \boldsymbol{R}_{\perp}=\mathbf{0}_{\perp}\right\rangle=\mathcal{N} \int \frac{\mathrm{d}^{2} \boldsymbol{p}_{\perp}}{(2 \pi)^{2}}\left|p^{+}, \boldsymbol{p}_{\perp}\right\rangle$

where $\left|p^{+}, \boldsymbol{p}_{\perp}\right\rangle$ are the light-cone helicity eigenstates. $\mathcal{N}$ is a normalization factor, $\boldsymbol{R}_{\perp}$ is the center of transverse momentum, which is defined as

$\boldsymbol{R}_{\perp}=\frac{\sum_{i} x_{i} \boldsymbol{b}_{\perp, i}}{\sum_{i} x_{i}}=\sum_{i} x_{i} \boldsymbol{b}_{\perp, i}$,

where $\boldsymbol{b}_{\perp}$ is the distance from the center of transverse momentum, and we sum over all partons in the hadron.

The impact parameter dependent PDFs are given by

$$
\begin{aligned}
q\left(x, \boldsymbol{b}_{\perp}^{2}\right) \\
=\int \frac{\mathrm{d} x^{-}}{4 \pi} e^{i x p^{+} x^{-}}\left\langle p^{+}, \mathbf{0}_{\perp}\right| \bar{q}\left(-\frac{x^{-}}{2}, \boldsymbol{b}_{\perp}\right) \gamma^{+} q \\
\quad \times\left(\frac{x^{-}}{2}, \boldsymbol{b}_{\perp}\right)\left|p^{+}, \mathbf{0}_{\perp}\right\rangle \\
=|\mathcal{N}|^{2} \int \frac{\mathrm{d}^{2} \boldsymbol{p}_{\perp}}{(2 \pi)^{2}} \int \frac{\mathrm{d}^{2} \boldsymbol{p}_{\perp}^{\prime}}{(2 \pi)^{2}} \int \frac{\mathrm{d} x^{-}}{4 \pi} e^{i x p^{+} x^{-}} e^{i \boldsymbol{b}_{\perp} \cdot\left(\boldsymbol{p}_{\perp}-\boldsymbol{p}_{\perp}^{\prime}\right)} \\
\quad \times\left\langle p^{+}, \boldsymbol{p}_{\perp}^{\prime}\left|\bar{q}\left(-\frac{x^{-}}{2}, \mathbf{0}_{\perp}\right) \gamma^{+} q\left(\frac{x^{-}}{2}, \mathbf{0}_{\perp}\right)\right| p^{+}, \boldsymbol{p}_{\perp}\right\rangle \\
=\int \frac{\mathrm{d}^{2} \boldsymbol{q}_{\perp}}{(2 \pi)^{2}} e^{-i \boldsymbol{b}_{\perp} \cdot \boldsymbol{q}_{\perp} H^{q}}\left(x, 0,-\boldsymbol{q}_{\perp}^{2}\right) .
\end{aligned}
$$

This means that the impact parameter dependent PDFs defined above are the Fourier transform of $H^{u}\left(x, 0,-\boldsymbol{q}_{\perp}^{2}\right)$, therefore, when we get $H^{u}\left(x, 0,-\boldsymbol{q}_{\perp}^{2}\right)$, parton distribution as a function of the distance $\boldsymbol{b}_{\perp}$ and the light-cone momentum fraction $x$ can be determined.
When $\xi \rightarrow 0$ and $t \neq 0$, the GPDs become

$$
\begin{aligned}
H^{u}\left(x, 0,-\boldsymbol{q}_{\perp}^{2}\right)= & \frac{3 Z_{K}}{4 \pi^{2}} \overline{\mathcal{C}}_{1}\left(\sigma_{1}\right) \\
& +\frac{3 Z_{K}}{4 \pi^{2}} \int_{0}^{1-x} \mathrm{~d} \alpha \frac{1}{\sigma_{8}} \overline{\mathcal{C}}_{2}\left(\sigma_{8}\right) \\
& \times\left((x-1) \boldsymbol{q}_{\perp}^{2}+2 x\left(m_{K}^{2}-\left(M_{u}-M_{s}\right)^{2}\right)\right),(40) \\
E^{u}\left(x, 0,-\boldsymbol{q}_{\perp}^{2}\right)= & \frac{N_{c} Z_{K}}{2 \pi^{2}} \\
& \times \int_{0}^{1-x} \mathrm{~d} \alpha m_{K}\left(\left(M_{s}-M_{u}\right) \alpha+M_{u}\right) \frac{\overline{\mathcal{C}}_{2}\left(\sigma_{8}\right)}{\sigma_{8}},
\end{aligned}
$$

where $x$ is defined in the region $x \in[0,1]$, then we can get

$$
\begin{aligned}
& u_{K}\left(x, \boldsymbol{b}_{\perp}^{2}\right)=\frac{N_{c} Z_{K}}{4 \pi^{2}} \int \frac{\mathrm{d}^{2} \boldsymbol{q}_{\perp}}{(2 \pi)^{2}} e^{-i \boldsymbol{b}_{\perp} \cdot \boldsymbol{q}_{\perp} \overline{\mathcal{C}}_{1}\left(\sigma_{1}\right)+\frac{N_{c} Z_{K}}{32 \pi^{3}} \int_{0}^{1-x} \mathrm{~d} \alpha} \\
& \quad \times \int \mathrm{d} \tau\left(\frac{(x-1)+2 \alpha \tau x(1-\alpha-x)\left(m_{K}^{2}-\left(M_{u}-M_{s}\right)^{2}\right)}{\alpha^{2} \tau^{2}(1-\alpha-x)^{2}}\right. \\
& \left.\quad+\frac{(x-1) \boldsymbol{b}_{\perp}^{2}}{4 \alpha^{3} \tau^{3}(1-\alpha-x)^{3}}\right) \\
& \quad \times e^{-\tau\left((1-x) M_{u}^{2}+x M_{s}^{2}-x(1-x) m_{K}^{2}\right)} e^{-\frac{b_{\perp}^{2}}{4 \tau \alpha(1-\alpha-x)}}, \\
& u_{K}^{T}\left(x, \boldsymbol{b}_{\perp}^{2}\right)=\frac{N_{c} Z_{K}}{16 \pi^{3}} \\
& \times \int_{0}^{1-x} \mathrm{~d} \alpha \int \mathrm{d} \tau \frac{m_{K}\left(\left(M_{s}-M_{u}\right) \alpha+M_{u}\right)}{\alpha(1-\alpha-x) \tau} \\
& \quad \times e^{-\frac{1}{4 \tau(1-\alpha-x) \alpha} \boldsymbol{b}_{\perp}^{2}} e^{-\tau\left((1-x) M_{u}^{2}+x M_{s}^{2}-x(1-x) m_{K}^{2}\right)},
\end{aligned}
$$

when integrating $\boldsymbol{b}_{\perp}$ we can get the $u$ quark PDF in Eq. (24).

We hope $H^{u}\left(x, 0,-\boldsymbol{q}_{\perp}^{2}\right)$ becomes independent of $-\boldsymbol{q}_{\perp}^{2}$ as $x \rightarrow 1$. It should be highlighted that this behavior is in accordance with "Feynman picture" for hadron form factors approximating $x=1$. In this picture, when one parton carries $x \approx 1$ and all others are "tiny" so that they can be easily scattered elastically, because the wee partons do not have a direction and therefore can be easily "sheer off". Thus, in the Feynman picture, the active quark takes a larger fraction of the hardron's overall momentum contributing to the FF and we have the tendency to a weaker $t$ dependence [96].

The width distribution of partons in the kaon for a given momentum fraction $x$ is

$\left\langle\boldsymbol{r}_{\perp}^{2}\right\rangle_{x}=\frac{\int \mathrm{d}^{2} \boldsymbol{b}_{\perp} \boldsymbol{b}_{\perp}^{2} q_{K}\left(x, \boldsymbol{b}_{\perp}^{2}\right)}{\int \mathrm{d}^{2} \boldsymbol{b}_{\perp} q_{K}\left(x, \boldsymbol{b}_{\perp}^{2}\right)}$,

when $x \rightarrow 1$, this impact parameter should be zero. This is because the struck quark becomes nearer to the center of momentum with the increasing of its weight. For our results, $\int \mathrm{d}^{2} \boldsymbol{b}_{\perp} u_{K}\left(x, \boldsymbol{b}_{\perp}^{2}\right)$ is the $u$ quark PDF in Eq. (24), when $x \rightarrow$ 1 ,

$u_{K}(1)=\frac{3 Z_{K}}{4 \pi^{2}} \overline{\mathcal{C}}_{1}\left(M_{s}^{2}\right)$ 


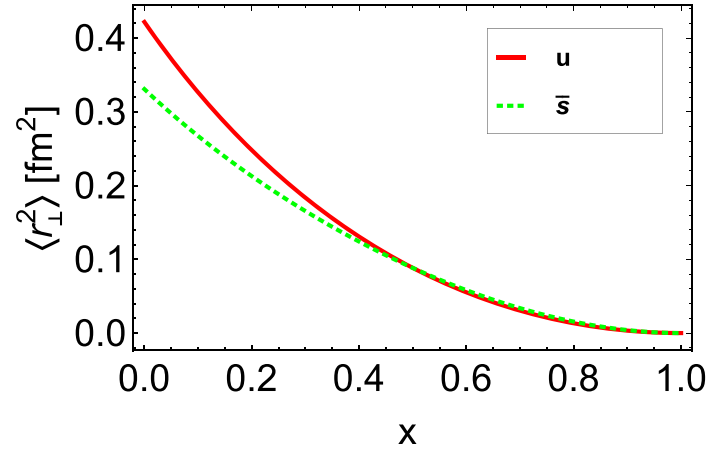

Fig. 8 The width of the distribution of partons in the kaon for a given momentum fraction $x$ defined in Eq. (44)

it is a definite value. For $\int \mathrm{d}^{2} \boldsymbol{b}_{\perp} \boldsymbol{b}_{\perp}^{2} u_{K}\left(x, \boldsymbol{b}_{\perp}^{2}\right)$, for the first term of Eq. (42),

$$
\begin{gathered}
\int \mathrm{d}^{2} \boldsymbol{b}_{\perp} \boldsymbol{b}_{\perp}^{2} \frac{N_{c} Z_{K}}{4 \pi^{2}} \int \frac{\mathrm{d}^{2} \boldsymbol{q}_{\perp}}{(2 \pi)^{2}} e^{-i \boldsymbol{b}_{\perp} \cdot \boldsymbol{q}_{\perp} \overline{\mathcal{C}}_{1}\left(\sigma_{1}\right)} \\
=\frac{N_{c} Z_{K}}{4 \pi^{2}} \int \mathrm{d}^{2} \boldsymbol{b}_{\perp} \boldsymbol{b}_{\perp}^{2} \delta^{2}\left(\boldsymbol{b}_{\perp}\right) \overline{\mathcal{C}}_{1}\left(\sigma_{1}\right)=0
\end{gathered}
$$

which means this term is zero, it is $x$ independent. The integration of the second term in Eq. (42) is restricted to the region $\alpha \in[0,1-x]$; when $x \rightarrow 1$, the integration will vanish, so $\int \mathrm{d}^{2} \boldsymbol{b}_{\perp} \boldsymbol{b}_{\perp}^{2} u_{K}\left(x, \boldsymbol{b}_{\perp}^{2}\right)$ vanishes when $x \rightarrow 1$. Our results satisfy $\left\langle\boldsymbol{r}_{\perp}^{2}\right\rangle_{x \rightarrow 1}=0$. This also can be seen from Fig. 8 , the width distribution of partons in the kaon $\left\langle\boldsymbol{r}_{\perp}^{2}\right\rangle_{x}$ become zero when $x \rightarrow 1$. From the diagram we can also see that in the small momentum fraction region, the $u$ quark is wider than the $s$ quark, with the increasing of $x$, the two lines almost coincide, which means the two quarks have almost the same width.

From Eq. (40) we can see that the first term is $-\boldsymbol{q}_{\perp}^{2}$ independent, for the second term, the range of the integration is $\alpha \in[0,1-x]$, when $x \rightarrow 1$, this term will vanish, so $H^{u}\left(x, 0,-\boldsymbol{q}_{\perp}^{2}\right)$ becomes $-\boldsymbol{q}_{\perp}^{2}$ independent, as expected.

The mean-squared $\boldsymbol{b}_{\perp}$ is defined as

$$
\left\langle\boldsymbol{b}_{\perp}^{2}\right\rangle=\int_{0}^{1} \mathrm{~d} x \int \mathrm{d}^{2} \boldsymbol{b}_{\perp} \boldsymbol{b}_{\perp}^{2} q_{K}\left(x, \boldsymbol{b}_{\perp}^{2}\right)
$$

then we obtain $\left\langle\boldsymbol{b}_{\perp}^{2}\right\rangle_{K}^{u}=0.149 \mathrm{fm}^{2},\left\langle\boldsymbol{b}_{\perp}^{2}\right\rangle_{K}^{s}=0.088 \mathrm{fm}^{2}$, for pion $\left\langle\boldsymbol{b}_{\perp}^{2}\right\rangle_{\pi}^{u}=0.139 \mathrm{fm}^{2}$. This means that in the kaon the $s$ quark is closer to the center of transverse momentum than the $u$ quark.

\subsection{Light-front transverse-spin distributions}

The light-front transverse-spin distributions are defined as [97]

$\rho^{n}\left(\boldsymbol{b}_{\perp}, \boldsymbol{s}_{\perp}\right)=\frac{1}{2}\left[A_{n, 0}\left(\boldsymbol{b}_{\perp}^{2}\right)-\frac{\varepsilon_{\perp}^{i j} \boldsymbol{s}_{\perp}^{i} \boldsymbol{b}_{\perp}^{j}}{m_{H}} B_{n, 0}^{\prime}\left(\boldsymbol{b}_{\perp}^{2}\right)\right]$,

where $m_{H}$ is the hadron mass, $B_{n, 0}^{\prime}\left(\boldsymbol{b}_{\perp}^{2}\right)=\partial_{\boldsymbol{b}_{\perp}^{2}} B_{n, 0}\left(\boldsymbol{b}_{\perp}^{2}\right)$, $A_{n, 0}\left(\boldsymbol{b}_{\perp}^{2}\right)$ and $B_{n, 0}\left(\boldsymbol{b}_{\perp}^{2}\right)$ are the two-dimensional Fourier transform of $A_{n, 0}\left(\boldsymbol{q}_{\perp}^{2}\right)$ and $B_{n, 0}\left(\boldsymbol{q}_{\perp}^{2}\right)$.

In Fig. 9, we plot the light-front transverse-spin distributions $\rho_{u}^{1}\left(\boldsymbol{b}_{\perp}, \boldsymbol{s}_{\perp}\right)$ and $\rho_{u}^{2}\left(\boldsymbol{b}_{\perp}, \boldsymbol{s}_{\perp}\right)$, when the quark is polarized in the light-front-transverse $+x$ direction, the transversespin density is not symmetric around $\boldsymbol{b}_{\perp}=\left(b_{x}=0, b_{y}=0\right)$ anymore, the peaks shift to $\left(b_{x}=0, b_{y}>0\right)$.

The average transverse shift is

$\left\langle b_{\perp}^{y}\right\rangle_{n}^{u}=\frac{\int \mathrm{d}^{2} \boldsymbol{b}_{\perp} b_{\perp}^{y} \rho_{u}^{n}\left(\boldsymbol{b}_{\perp}, \boldsymbol{s}_{\perp}\right)}{\int \mathrm{d}^{2} \boldsymbol{b}_{\perp} \rho_{u}^{n}\left(\boldsymbol{b}_{\perp}, \boldsymbol{s}_{\perp}\right)}=\frac{1}{2 m_{K}} \frac{B_{n, 0}^{u}(0)}{A_{n, 0}^{u}(0)}$

in the $y$ direction for a transverse quark spin $s_{\perp}=(1,0)$ in the $x$ direction. Our results give $\left\langle b_{\perp}^{y}\right\rangle_{1}^{u}=0.116 \mathrm{fm}$ and $\left\langle b_{\perp}^{y}\right\rangle_{2}^{u}=0.083 \mathrm{fm}$, which are larger than the pion results, but smaller compared with the lattice results in Ref. [97]. Their results for the pion are larger than our kaon results. That is because the NJL model is a contact interaction, FFs $A_{n, 0}^{u}\left(\boldsymbol{q}_{\perp}^{2}\right)$ and $B_{n, 0}^{u}\left(\boldsymbol{q}_{\perp}^{2}\right)$ are harder than the experimental results.

\section{Light-front wave functions}

As mentioned before, LFWFs offer the underlying connection between QCD and its fundamental degrees of freedom: quarks, gluons, and their asymptotic hadronic states. In phenomenology, LFWFs are particularly important because all hadronic properties can be deduced from them in principle. In this section, we will derive the lowest-order $q \bar{q}$ valence LFWF for the kaon. Using the properties of LFWF, we generate kaon PDF, PDA, decay constant, TMD, and GPD, and compare these distributions with those obtained from the vector GPD, respectively.

\subsection{The definition and calculation of the kaon LFWF}

In the NJL model, the Bethe-Salpeter vertex for the kaon has the form

$\Gamma_{K}\left(p^{\prime}, p\right)=\alpha_{1} \gamma_{5}+\alpha_{2} \not q \gamma_{5}$, 

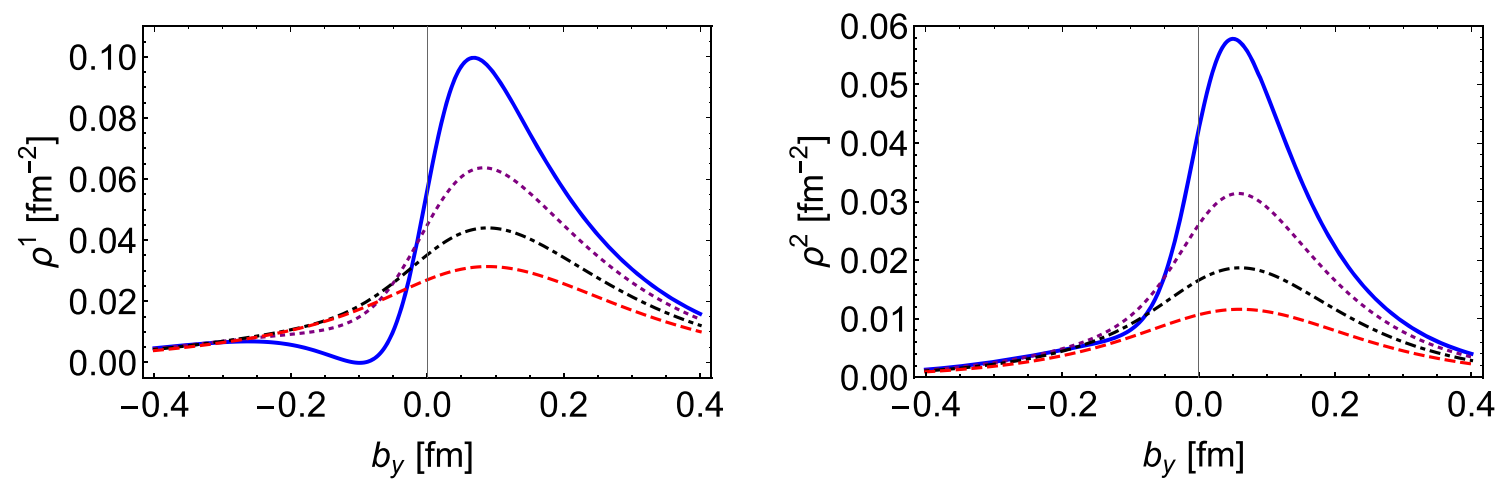

Fig. 9 The light-front transverse-spin distribution $\rho_{u}^{1}\left(\boldsymbol{b}_{\perp}, \boldsymbol{s}_{\perp}\right)$ and $\rho_{u}^{2}\left(\boldsymbol{b}_{\perp}, \boldsymbol{s}_{\perp}\right), \boldsymbol{s}_{\perp}=(1,0)$ at constant $b_{x}$ fm: $b_{x}=0.1 \mathrm{fm}-\mathrm{blue}$ solid curve, $b_{x}=0.15 \mathrm{fm}$ - purple dotted curve, $b_{x}=0.2 \mathrm{fm}$-black dot-dashed curve, $b_{x}=0.25 \mathrm{fm}$ - red dashed curve

and the kaon Bethe-Salpeter wave function is defined as

$\chi_{K}\left(k^{\prime}, k\right)=i S_{u}(k) \Gamma_{K}(q) i S_{s}(k-q)$.

$\varphi_{K}\left(x, \boldsymbol{k}_{\perp}\right)$ is defined as

$\varphi_{K}\left(x, \boldsymbol{k}_{\perp}\right)=\int \frac{\mathrm{d} k^{+} \mathrm{d} k^{-}}{2 \pi} \delta\left(x-\frac{k^{+}}{q^{+}}\right) \chi_{K}\left(k^{\prime}, k\right)$.

The kaon LFWF is defined in Refs. [98,99], Eq. (22) of Ref. [98]:

$$
\begin{aligned}
- & 2 q^{+} \sqrt{x \bar{x}} \psi_{K}\left(x, \boldsymbol{k}_{\perp}, \lambda, \lambda^{\prime}\right) \\
& =i \sqrt{6} \bar{u}_{u}\left(x q^{+}, \boldsymbol{k}_{\perp}, \lambda\right) \gamma^{+} \varphi_{K}\left(x, \boldsymbol{k}_{\perp}\right) \gamma^{+} v_{s}\left(\bar{x} q^{+},-\boldsymbol{k}_{\perp}, \lambda^{\prime}\right)
\end{aligned}
$$

where $\bar{x}=1-x$, the definition of the basis spinors $u$ and $v$ are defined in Ref. [99]

$$
\begin{aligned}
& u_{u}\left(x q^{+}, \boldsymbol{k}, \lambda\right)=\frac{1}{\sqrt{x q^{+}}}\left(x q^{+}+\beta M_{u}+\alpha_{i} k_{i}\right) X_{\lambda}, \\
& v_{s}\left((1-x) q^{+},-\boldsymbol{k}_{\perp}, \lambda^{\prime}\right) \\
& \quad=\frac{1}{\sqrt{q^{+}(1-x)}}\left((1-x) q^{+}-\beta M_{s}-\alpha_{i} k_{i}\right) X_{-\lambda^{\prime}},
\end{aligned}
$$

where

$$
\begin{aligned}
& X_{\lambda}=\frac{1}{\sqrt{2}}\left(\begin{array}{c}
\chi_{\lambda} \\
\lambda \chi_{\lambda}
\end{array}\right), \\
& \chi_{1}=\chi_{\uparrow}=\left(\begin{array}{l}
1 \\
0
\end{array}\right), \quad \chi_{-1}=\chi_{\downarrow}=\left(\begin{array}{l}
0 \\
1
\end{array}\right), \\
& \gamma^{0}=\left(\begin{array}{cc}
1 & 0 \\
0 & -1
\end{array}\right), \quad \gamma^{i}=\left(\begin{array}{cc}
0 & \sigma^{i} \\
-\sigma^{i} & 0
\end{array}\right), \quad \gamma^{5}=\left(\begin{array}{ll}
0 & 1 \\
1 & 0
\end{array}\right),
\end{aligned}
$$

where $\beta=\gamma_{0}, \alpha_{i}=\gamma^{0} \gamma^{i}, i=1$, 2. Using the relationships in Ref. [99]

$$
\begin{aligned}
& \bar{u}_{u}\left(q^{+} x, \boldsymbol{k}_{\perp}, \lambda\right) \gamma^{+}\left(\not k+M_{u}\right)=2 x q^{+} \bar{u}_{u}\left(q^{+} x, \boldsymbol{k}_{\perp}, \lambda\right), \\
& \left((\not k-\not q)+M_{s}\right) \gamma^{+} v_{s}\left(q^{+} \bar{x},-\boldsymbol{k}_{\perp}, \lambda^{\prime}\right) \\
& \quad=-2 \bar{x} q^{+} v_{s}\left(\bar{x} q^{+},-\boldsymbol{k}_{\perp}, \lambda^{\prime}\right) .
\end{aligned}
$$

The lowest-order $u$ quark LFWF of the kaon can be given by

$$
\begin{aligned}
& \psi_{2}(\left(x, \boldsymbol{k}_{\perp}, \lambda, \lambda^{\prime}\right) \\
&= i \sqrt{6} \int \frac{d k^{+} d k^{-}}{\pi} \delta\left(x-\frac{k^{+}}{q^{+}}\right) \frac{\sqrt{x \bar{x}}}{\left(k^{2}-M_{u}^{2}\right)\left((k-q)^{2}-M_{s}^{2}\right)} \\
& \quad \times \bar{u}_{u}\left(x q^{+}, \boldsymbol{k}_{\perp}, \lambda\right) \gamma^{+}\left(\alpha_{1} \gamma_{5}+\alpha_{2} \not \gamma_{5}\right) \gamma^{+} v_{s}\left(\bar{x} q^{+},-\boldsymbol{k}_{\perp}, \lambda^{\prime}\right),
\end{aligned}
$$

in the NJL model, $\alpha_{1}=\sqrt{Z_{K}}$, we do not consider the mixing of the pseudoscalar and the pseudovector interaction terms in the Bethe-Salpeter equation for the kaon, so $\alpha_{2}=0$; then we arrive at

$$
\begin{aligned}
\psi_{2}\left(x, \boldsymbol{k}_{\perp}, \lambda, \lambda^{\prime}\right)=\left(\begin{array}{ll}
\psi_{2 \uparrow \uparrow} & \psi_{2 \uparrow \downarrow} \\
\psi_{2 \downarrow \uparrow} & \psi_{2 \downarrow \downarrow}
\end{array}\right) \\
=\frac{\sqrt{6} \sqrt{Z_{K}}}{\boldsymbol{k}_{\perp}^{2}+(1-x) M_{u}^{2}+x M_{s}^{2}+m_{K}^{2}(x-1) x} \\
\quad \times\left(\begin{array}{cc}
-k_{-\lambda} & \left(M_{u}+x\left(M_{s}-M_{u}\right)\right) \\
-\left(M_{u}+x\left(M_{S}-M_{u}\right)\right) & -k_{-\lambda^{\prime}}
\end{array}\right),
\end{aligned}
$$

where $\lambda$ is the quark spin, $k_{\lambda} \equiv k_{1}+i \lambda k_{2}, \psi_{2 \uparrow \uparrow}$ and $\psi_{2 \downarrow \downarrow}$ is the spin parallel to $S_{z}= \pm 1, \psi_{2 \uparrow \downarrow}$ and $\psi_{2 \downarrow \uparrow}$ is the spin anti-parallel $S_{z}=0$. For the $\psi_{2 \uparrow \uparrow}$ term $\lambda=1$, for the $\psi_{2 \downarrow \downarrow}$ term $\lambda^{\prime}=-1$. Both spin alignments should contribute to the kaon wave function, which was pointed out by Leutwyler $[100,101]$. The LFWF can be reduced to

$\psi_{2}\left(x, \boldsymbol{k}_{\perp}, \lambda, \lambda^{\prime}\right)$ 


$$
\begin{aligned}
= & \frac{\sqrt{6} \sqrt{Z_{K}}}{\boldsymbol{k}_{\perp}^{2}+(1-x) M_{u}^{2}+x M_{s}^{2}+m_{K}^{2}(x-1) x} \\
& \times\left(\begin{array}{cc}
-\left(k_{1}-i k_{2}\right) & \left(M_{u}+x\left(M_{s}-M_{u}\right)\right) \\
-\left(M_{u}+x\left(M_{s}-M_{u}\right)\right) & -\left(k_{1}+i k_{2}\right)
\end{array}\right) .
\end{aligned}
$$

In the following calculation, we will use the PTR scheme.

\subsection{The properties of the kaon LFWF}

\subsubsection{Normalization}

The normalization condition is

$\sum_{\lambda, \lambda^{\prime}} \int_{0}^{1} \mathrm{~d} x \int \frac{\mathrm{d}^{2} \boldsymbol{k}_{\perp}}{16 \pi^{3}}\left|\psi_{2}\left(x, \boldsymbol{k}_{\perp}, \lambda, \lambda^{\prime}\right)\right|^{2}=1$,

this means the probability of finding kaon in its valence state is 1 . We obtain

$$
\begin{aligned}
\frac{1}{Z_{K}}= & \frac{3}{4 \pi^{2}} \int_{0}^{1} \mathrm{~d} x \overline{\mathcal{C}}_{1}\left(\sigma_{1}\right) \\
& +\frac{3}{2 \pi^{2}} \int_{0}^{1} \mathrm{~d} x x(1-x)\left(m_{K}^{2}-\left(M_{u}-M_{s}\right)^{2}\right) \frac{\overline{\mathcal{C}}_{2}\left(\sigma_{1}\right)}{\sigma_{1}}
\end{aligned}
$$

where $Z_{K}=20.47$, the same as $Z_{K}$ obtained from Eq. (8).

\subsubsection{Kaon decay constant}

Similar to the pion, the constraint offered by the kaon decay constant $f_{K}$ on the wave function is

$\int_{0}^{1} \mathrm{~d} x \int \frac{\mathrm{d}^{2} \boldsymbol{k}_{\perp}}{16 \pi^{3}} \psi_{2 \uparrow \downarrow}\left(x, \boldsymbol{k}_{\perp}\right)=\frac{f_{K}}{2 \sqrt{6}}$,

for our result

$$
\begin{aligned}
f_{K} & =\frac{3 \sqrt{Z_{K}}}{4 \pi^{2}} \int_{0}^{1} \mathrm{~d} x\left(M_{u}+x\left(M_{s}-M_{u}\right)\right) \overline{\mathcal{C}}_{1}\left(\sigma_{1}\right) \\
& =0.092 \mathrm{GeV} .
\end{aligned}
$$

\subsubsection{Constraint from $K^{0} \rightarrow 2 \gamma$}

This constraint assumes the very simple form

$$
\begin{aligned}
& \int_{0}^{1} \mathrm{~d} x \psi_{2 \uparrow \downarrow}\left(x, \mathbf{0}_{\perp}\right) \\
& \quad=\int_{0}^{1} \mathrm{~d} x \frac{\sqrt{6} \sqrt{Z_{K}}\left(M_{u}+x\left(M_{s}-M_{u}\right)\right)}{\left((1-x) M_{u}^{2}+x M_{s}^{2}+m_{K}^{2}(x-1) x\right)}=\frac{\sqrt{6}}{f_{K}}
\end{aligned}
$$

and we obtain $f_{K}=0.094 \mathrm{GeV}$.

\subsubsection{Kaon form factor}

The kaon electromagnetic form factor is given by the overlap of two wave functions,

$$
\begin{aligned}
F_{K}^{u}\left(\boldsymbol{q}_{\perp}^{2}\right)= & \sum_{\lambda, \lambda^{\prime}} \int_{0}^{1} \mathrm{~d} x \int \frac{\mathrm{d}^{2} \boldsymbol{k}_{\perp}}{16 \pi^{3}} \\
& \times \psi_{2}^{\dagger}\left(x, \boldsymbol{k}_{\perp}^{\prime}, \lambda, \lambda^{\prime}\right) \psi_{2}\left(x, \boldsymbol{k}_{\perp}, \lambda, \lambda^{\prime}\right),
\end{aligned}
$$

where $\boldsymbol{k}_{\perp}^{\prime}=\boldsymbol{k}_{\perp}+(1-x) \boldsymbol{q}_{\perp}$ is the transverse momentum of the struck quark. We have

$$
\begin{aligned}
F_{K}^{u}\left(\boldsymbol{q}_{\perp}^{2}\right)= & \frac{3 Z_{K}}{4 \pi^{2}} \int_{0}^{1} \mathrm{~d} x \overline{\mathcal{C}}_{1}\left(\sigma_{1}\right) \\
& +\frac{3 Z_{K}}{4 \pi^{2}} \int_{0}^{1} \mathrm{~d} x \int_{0}^{1} \mathrm{~d} z \frac{1}{\sigma_{9}} \overline{\mathcal{C}}_{2}\left(\sigma_{9}\right) \\
& \times\left(2 x(1-x)\left(m_{K}^{2}-\left(M_{u}-M_{s}\right)^{2}\right)-(1-x)^{2} \boldsymbol{q}_{\perp}^{2}\right),
\end{aligned}
$$

comparing with the result in Eq. (31), it is hard to see their relation only through the equations; instead, we have checked that they are exactly the same numerically.

The kaon charge radius $r_{K}$ uses the Drell-Yan formula, which is given by the gradient of form factor at zero momentum transfer,

$F_{K}\left(\boldsymbol{q}_{\perp}^{2}\right) \equiv 1-\frac{r_{K}^{2}}{6} \boldsymbol{q}_{\perp}^{2}+O\left(\boldsymbol{q}_{\perp}^{4}\right)$,

the charge radius $r_{K}$ is defined as

$\left\langle r_{K}^{2}\right\rangle=-\left.6 \frac{\partial F_{K}\left(\boldsymbol{q}_{\perp}^{2}\right)}{\partial \boldsymbol{q}_{\perp}^{2}}\right|_{\boldsymbol{q}_{\perp}^{2}=0}$,

and we obtain $r_{K}^{u}=0.477 \mathrm{fm}, r_{K}^{s}=0.363 \mathrm{fm}$.

\subsubsection{Transverse size}

The r.m.s. transverse momentum is given by

$$
\begin{aligned}
\left\langle\boldsymbol{k}_{\perp}^{2}\right\rangle= & \sum_{\lambda, \lambda^{\prime}} \int_{0}^{1} \mathrm{~d} x \int \frac{\mathrm{d}^{2} \boldsymbol{k}_{\perp}}{16 \pi^{3}} \boldsymbol{k}_{\perp}^{2}\left|\psi_{2}\left(x, \boldsymbol{k}_{\perp}, \lambda, \lambda^{\prime}\right)\right|^{2} \\
= & \frac{3 Z_{K}}{4 \pi^{2}} \int_{0}^{1} \mathrm{~d} x \mathcal{C}_{0}\left(\sigma_{1}\right) \\
& +\frac{3 Z_{K}}{4 \pi^{2}} \int_{0}^{1} \mathrm{~d} x x(1-x)\left(m_{K}^{2}-\left(M_{u}-M_{s}\right)^{2}\right) \overline{\mathcal{C}}_{1}\left(\sigma_{1}\right) \\
= & (0.486 \mathrm{GeV})^{2},
\end{aligned}
$$

which gives a transverse size scale $R_{\perp}$ :

$R_{\perp}^{2} \equiv \frac{1}{\left\langle\boldsymbol{k}_{\perp}^{2}\right\rangle}=(0.405 \mathrm{fm})^{2}$ 
which is slightly smaller than the charge radius $r_{K}^{u}$. The dimensionless quantity $C=f_{K} R_{\perp}$ [102] connects the "core radius" $R_{\perp}$ with the decay constant $f_{K}$. Our result is $C=0.189$, this is coincident with the result $C \simeq 0.2$ in Ref. [103].

\subsubsection{Kaon structure function}

The valence structure function of the kaon is given by the squares of the LFWF:

$$
\begin{aligned}
u_{K}(x)= & \sum_{\lambda, \lambda^{\prime}} \int \frac{\mathrm{d}^{2} \boldsymbol{k}_{\perp}}{16 \pi^{3}}\left|\psi_{2}\left(x, \boldsymbol{k}_{\perp}, \lambda, \lambda^{\prime}\right)\right|^{2} \\
= & \frac{3 Z_{K}}{4 \pi^{2}} \overline{\mathcal{C}}_{1}\left(\sigma_{1}\right) \\
& +\frac{3 Z_{K}}{2 \pi^{2}} x(1-x)\left(m_{K}^{2}-\left(M_{u}-M_{s}\right)^{2}\right) \frac{\overline{\mathcal{C}}_{2}\left(\sigma_{1}\right)}{\sigma_{1}},
\end{aligned}
$$

when integrating $x$ we get the normalization condition, which means that the probability to find a valence quark in the kaon is unity, as it should. For the mean value of the momentum fraction $x$ carried by the $u$ quark

$$
\langle x\rangle^{u}=\int_{0}^{1} x u_{K}(x) \mathrm{d} x=0.431
$$

thus we have $\langle x\rangle^{S}=0.569$. This is different from the pion value $1 / 2$ [9], which means that the quarks do not share an equal amount of longitudinal momentum because of the difference of light quark and strange quark.

In the NJL model, in the chiral limit $m_{\pi}=0$, the pion PDF $q(x)=1[5,9,104]$. When $m_{\pi} \neq 0$, our results show the pion $q(x) \approx 1$, it fluctuates around 1 . Reference [5] plotted the pion PDF with different values of $m_{\pi}$. For comparison, we plot the diagrams of the $u$ quark PDFs of pion and kaon with different values of $m_{\pi}$ and $m_{K}$ in Fig. 10. From the diagrams we can see that different from the pion PDFs, the kaon PDFs do not show symmetry any more, the peaks shift to $x<0.5$.

\subsubsection{Parton distribution amplitude}

The PDA was introduced to describe the hard exclusive processes. The PDA from LFWF is defined as

$$
\begin{aligned}
\phi_{K}^{u}(x) & =\frac{2 \sqrt{6}}{f_{K}} \int \frac{\mathrm{d}^{2} \boldsymbol{k}_{\perp}}{16 \pi^{3}} \psi_{2 \uparrow \downarrow}\left(x, \boldsymbol{k}_{\perp}\right) \\
& =\frac{3 \sqrt{Z_{K}}}{4 \pi^{2} f_{K}}\left(M_{u}+x\left(M_{s}-M_{u}\right)\right) \overline{\mathcal{C}}_{1}\left(\sigma_{1}\right)
\end{aligned}
$$

and satisfies the relationship

$$
\int_{0}^{1} \mathrm{~d} x \phi_{K}^{u}(x)=1
$$

The pion PDA, in the chiral limit, similar to the pion PDF, is $\phi_{\pi}^{u}(x)=1$, when $m_{\pi} \neq 0$, it fluctuates around 1 , but the kaon is different. We do not plot the diagrams of the comparison pion and kaon PDAs because they are similar to the pion and kaon PDFs diagrams in Fig. 10, the peaks of the kaon PDAs shift to $x<0.5$.

\subsubsection{Transverse momentum dependent parton distribution}

The unpolarized $u$ quark TMDs of the kaon is given by

$$
\begin{aligned}
f_{K}^{u}\left(x, \boldsymbol{k}_{\perp}^{2}\right)= & \frac{1}{16 \pi^{3}} \sum_{\lambda, \lambda^{\prime}}\left|\psi_{2}\left(x, \boldsymbol{k}_{\perp}, \lambda, \lambda^{\prime}\right)\right|^{2} \\
= & \frac{3 Z_{K}}{2 \pi^{3}} \frac{1}{\sigma_{11}} \overline{\mathcal{C}}_{2}\left(\sigma_{11}\right) \\
& +\frac{3 Z_{K}}{4 \pi^{3}} x(1-x)\left(m_{K}^{2}-\left(M_{u}-M_{s}\right)^{2}\right) \\
& \times \frac{6}{\sigma_{11}^{2}} \overline{\mathcal{C}}_{3}\left(\sigma_{11}\right),
\end{aligned}
$$

when integrating $\boldsymbol{k}_{\perp}$, we get the $u$ quark PDF in Eq. (24). The $s$ quark TMD in kaon can be simply obtained from the $u$ quark distribution by momentum conservation,

$f_{K}^{u}\left(x, \boldsymbol{k}_{\perp}^{2}\right)=f_{K}^{s}\left(1-x, \boldsymbol{k}_{\perp}^{2}\right)$,

the quark TMDs of the kaon are plotted in Fig. 11.

\subsubsection{Generalized parton distribution}

The relationship between LFWF and GPD in the region $\xi \leq$ $x \leq 1$ is given by Ref. [105]:

$H(x, \xi, t)=\sum_{\lambda^{\prime} \lambda} \int \frac{\mathrm{d}^{2} \boldsymbol{k}_{\perp}}{16 \pi^{3}} \psi_{2}^{\dagger}\left(x^{\prime \prime}, \boldsymbol{k}_{\perp}^{\prime \prime}, \lambda^{\prime}\right) \psi_{2}\left(x^{\prime}, \boldsymbol{k}_{\perp}^{\prime}, \lambda\right)$,

where

$x^{\prime \prime}=\frac{x-\xi}{1-\xi}, \quad \boldsymbol{k}_{\perp}^{\prime \prime}=\boldsymbol{k}_{\perp}+\frac{1-x}{1-\xi} \frac{\boldsymbol{q}_{\perp}}{2}$,
$x^{\prime}=\frac{x+\xi}{1+\xi}, \quad \boldsymbol{k}_{\perp}^{\prime}=\boldsymbol{k}_{\perp}-\frac{1-x}{1+\xi} \frac{\boldsymbol{q}_{\perp}}{2}$,

one can obtain

$$
H^{u}(x, \xi, t)=\frac{3 Z_{K}}{8 \pi^{2}} \theta_{\xi 1} \overline{\mathcal{C}}_{1}\left(\sigma_{3}\right)+\frac{3 Z_{K}}{8 \pi^{2}} \theta_{\xi 1} \overline{\mathcal{C}}_{1}\left(\sigma_{4}\right)
$$



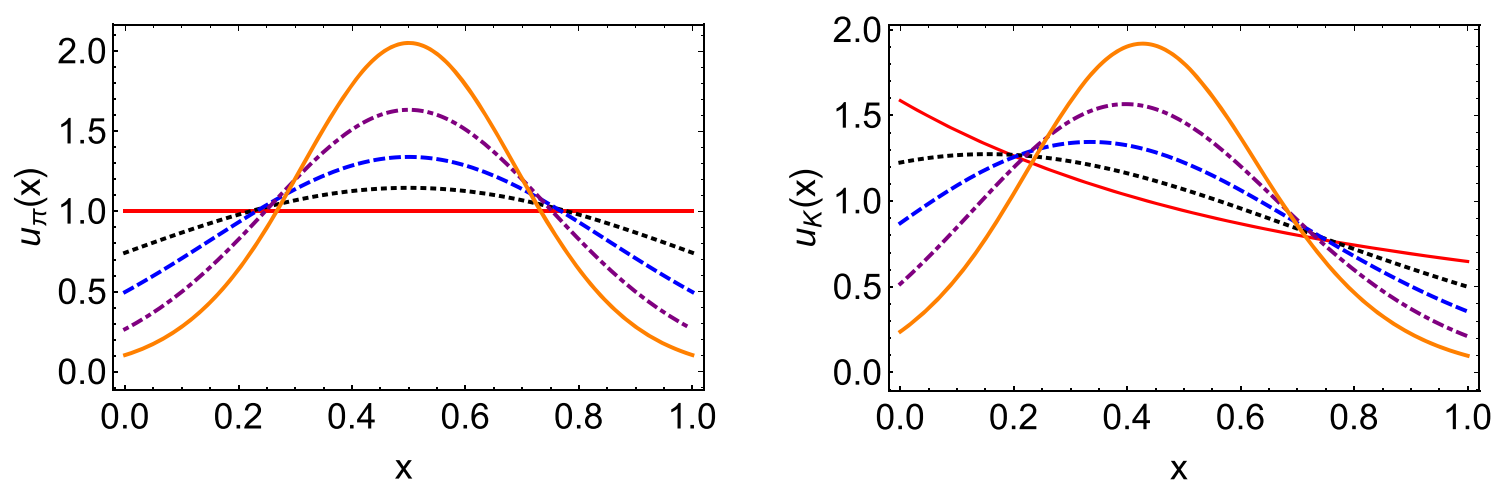

Fig. 10 Pion and kaon $u$ quark PDF with different values of $m_{\pi}$ and $m_{K}$ : thin red line $-m_{\pi}\left(m_{K}\right)=0 \mathrm{GeV}$, black dotted line $-m_{\pi}\left(m_{K}\right)=0.4$ $\mathrm{GeV}$, blue dashed line $-m_{\pi}\left(m_{K}\right)=0.6 \mathrm{GeV}$, purple dotdashed line $-m_{\pi}\left(m_{K}\right)=0.8 \mathrm{GeV}$, thick orange line $-m_{\pi}\left(m_{K}\right)=1.0 \mathrm{GeV}$
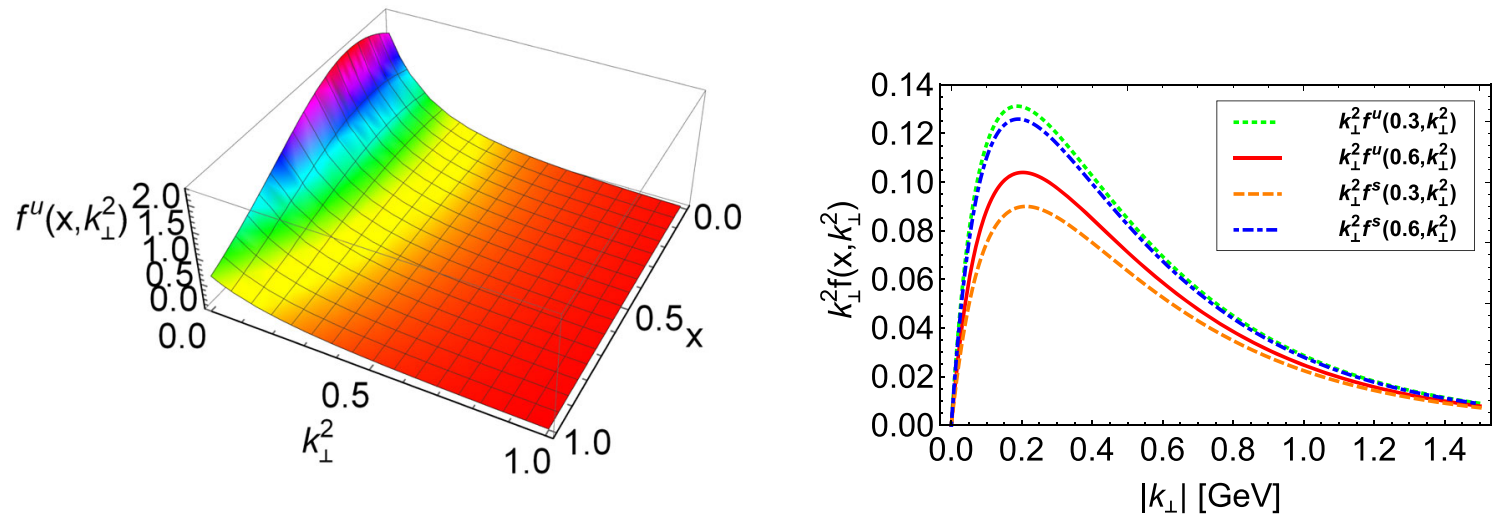

Fig. 11 Left panel $-f\left(x, k_{\perp}^{2}\right) u$ quark TMD of the kaon; and right panel $-k_{\perp}^{2} f\left(x, k_{\perp}^{2}\right)$ for $u$ and $\bar{s}$ quark of the kaon with different $x$

$$
\begin{aligned}
& -\frac{3 Z_{K}}{4 \pi^{2}} \int_{0}^{1} \mathrm{~d} z \theta_{\xi 1}(1-x)\left(\frac{2 x\left(M_{s}-M_{u}\right)^{2}}{(1+\xi)(1-\xi)}\right. \\
& \left.-m_{K}^{2}\left(\frac{(x-\xi)}{(1-\xi)^{2}}+\frac{(\xi+x)}{(\xi+1)^{2}}\right)-\frac{(1-x) t}{(\xi+1)^{2}(1-\xi)^{2}}\right) \\
& \times \frac{\overline{\mathcal{C}}_{2}\left(\sigma_{10}\right)}{\sigma_{10}} .
\end{aligned}
$$

In the forward limit $\xi=0$ and $t=0$ we get the quark PDF in Eq. (24), when integrating over $x$ we get the FF in Eq. (68).

\subsubsection{Mass distribution from LFWF GPD}

When $\xi=0$, the GPD becomes

$$
\begin{aligned}
H^{u}\left(x, 0,-\boldsymbol{q}_{\perp}^{2}\right)= & \frac{3 Z_{K}}{4 \pi^{2}} \overline{\mathcal{C}}_{1}\left(\sigma_{1}\right)+\frac{3 Z_{K}}{4 \pi^{2}} \int_{0}^{1} \mathrm{~d} z \frac{1}{\sigma_{9}} \overline{\mathcal{C}}_{2}\left(\sigma_{9}\right) \\
& \times(1-x)\left(2 x\left(m_{K}^{2}-\left(M_{s}-M_{u}\right)^{2}\right)\right. \\
& \left.-(1-x) \boldsymbol{q}_{\perp}^{2}\right),
\end{aligned}
$$

in the region $x \in[0,1]$, we examined that it is the same as Eq. (40), so we will get the same impact parameter dependent PDF $u_{K}\left(x, b_{\perp}^{2}\right)$ in Eq. (42).

The $u$ quark mass distribution is

$$
\begin{aligned}
A_{2,0}^{u}= & \int_{0}^{1} \mathrm{~d} x x H^{u}\left(x, 0,-\boldsymbol{q}_{\perp}^{2}\right) \\
= & \frac{3 Z_{K}}{4 \pi^{2}} \int_{0}^{1} \mathrm{~d} x x \overline{\mathcal{C}}_{1}\left(\sigma_{1}\right) \\
& +\frac{3 Z_{K}}{4 \pi^{2}} \int_{0}^{1} \mathrm{~d} z \int_{0}^{1} \mathrm{~d} x \frac{1}{\sigma_{9}} \overline{\mathcal{C}}_{2}\left(\sigma_{9}\right) \\
& \times x(1-x)\left(2 x\left(m_{K}^{2}-\left(M_{s}-M_{u}\right)^{2}\right)-(1-x) \boldsymbol{q}_{\perp}^{2}\right),
\end{aligned}
$$

in Fig. 12, we compare this with $A_{2,0}^{u}$ in Eq. (33). From the diagram, we can see that it is exactly the same, so we get the same light-cone energy radius and light-cone charge radius from the two methods. 


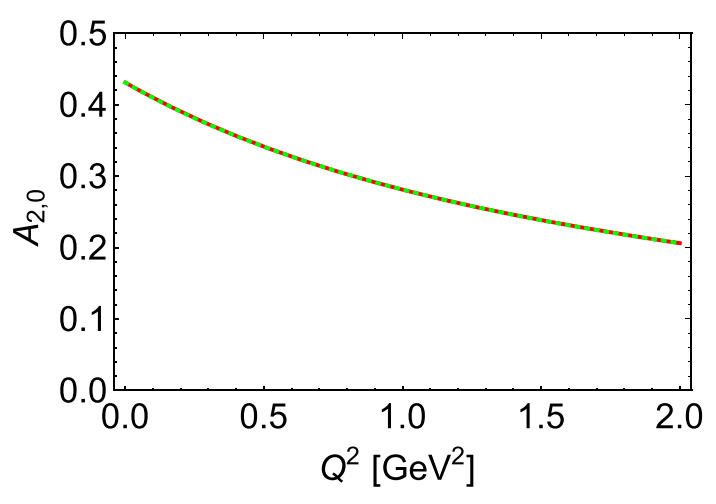

Fig. 12 The quark mass distribution $A_{2,0}$ from the two different methods: the red solid curve - Eq. (33) from vector GPD, the green dotted curve - Eq. (83) from LFWF and one obtains

$$
\begin{aligned}
W^{U U} & \left(x, \boldsymbol{k}_{\perp}, \boldsymbol{q}_{\perp}\right) \\
= & \frac{3 Z_{K}}{2 \pi^{3}} \frac{1}{\sigma_{11}} \overline{\mathcal{C}}_{2}\left(\sigma_{11}\right) \\
& -\frac{3 Z_{K}}{8 \pi^{3}} \int_{0}^{1} \mathrm{~d} z \frac{6}{\sigma_{12}^{2}} \overline{\mathcal{C}}_{3}\left(\sigma_{12}\right) \\
& \times(1-x)\left(2 x\left(\left(M_{s}-M_{u}\right)^{2}-m_{K}^{2}\right)+(1-x) \boldsymbol{q}_{\perp}^{2}\right) .
\end{aligned}
$$

Using our LFWF, we get the following expression of the $u$ quark unpolarized Wigner distribution of the kaon:

$$
\begin{aligned}
\rho_{U U}\left(x, \boldsymbol{k}_{\perp}, \boldsymbol{b}_{\perp}\right)= & \frac{3 Z_{K}}{2 \pi^{3}} \int \frac{\mathrm{d}^{2} \boldsymbol{q}_{\perp}}{(2 \pi)^{2}} e^{-i \boldsymbol{b}_{\perp} \cdot \boldsymbol{q}_{\perp}} \frac{1}{\sigma_{11}} \overline{\mathcal{C}}_{2}\left(\sigma_{11}\right) \\
& -\frac{3 Z_{K}}{32 \pi^{4}} \int_{0}^{1} \mathrm{~d} z \int \mathrm{d} \tau e^{-\tau\left(\boldsymbol{k}_{\perp}^{2}+(1-x) M_{u}^{2}+x M_{s}^{2}+m_{K}^{2}(x-1) x\right) e^{-\frac{1}{4 \tau z(1-z)(1-x)^{2}} \boldsymbol{b}_{\perp}^{2}}} \\
& \times \frac{\boldsymbol{b}_{\perp}^{2}+8\left(\left(M_{s}-M_{u}\right)^{2}-m_{K}^{2}\right) \tau^{2} x(x-1)^{3}(z-1)^{2} z^{2}+4 \tau(x-1)^{2}(z-1) z}{4 \tau^{2}(x-1)^{4}(z-1)^{3} z^{3}},
\end{aligned}
$$

\subsubsection{Wigner distribution}

The definition of the unpolarized Wigner distribution [106117], according to Refs. [74,117], is

$\rho_{U U}\left(x, \boldsymbol{k}_{\perp}, \boldsymbol{b}_{\perp}\right)=\int \frac{\mathrm{d}^{2} \boldsymbol{q}_{\perp}}{(2 \pi)^{2}} e^{-i \boldsymbol{b}_{\perp} \cdot \boldsymbol{q}_{\perp}} W^{U U}\left(x, \boldsymbol{k}_{\perp}, \boldsymbol{q}_{\perp}\right)$,

where $\boldsymbol{b}_{\perp}$ is the transverse momentum transfer, the Wigner correlator is given by

$$
\begin{aligned}
& W^{U U}\left(x, \boldsymbol{k}_{\perp}, \boldsymbol{q}_{\perp}\right) \\
& =\sum_{\lambda^{\prime} \lambda} \frac{1}{16 \pi^{3}} \psi_{2}^{\dagger}\left(x, \boldsymbol{k}_{\perp}^{\prime \prime}, \lambda^{\prime}\right) \psi_{2}\left(x, \boldsymbol{k}_{\perp}^{\prime}, \lambda\right),
\end{aligned}
$$

where

$$
\begin{aligned}
& \boldsymbol{k}_{\perp}^{\prime \prime}=\boldsymbol{k}_{\perp}+(1-x) \frac{\boldsymbol{q}}{2}, \\
& \boldsymbol{k}_{\perp}^{\prime}=\boldsymbol{k}_{\perp}-(1-x) \frac{\boldsymbol{q}}{2},
\end{aligned}
$$

for $\rho_{U U}\left(x, \boldsymbol{k}_{\perp}, \boldsymbol{b}_{\perp}\right)$ when integrating $\boldsymbol{k}_{\perp}$ we obtain Eq. (42), when integrating $\boldsymbol{b}_{\perp}$ we can get TMD in Eq. (77). We plot the unpolarized Wigner distribution in Fig. 13. The diagrams show that, firstly, we have a sharp peak at $\left(x=1, \boldsymbol{k}_{\perp}=\right.$ $\mathbf{0}, \boldsymbol{b}_{\perp}=\mathbf{0}$ ); secondly, the Wigner distribution shows a powerlaw suppression as $\boldsymbol{k}_{\perp}^{2}$ and/or $\boldsymbol{b}_{\perp}^{2}$ increasing; thirdly, it is negative on a neighborhood ( $x \simeq 0, \boldsymbol{k}_{\perp}^{2} \simeq 0$ ). The calculation of the unpolarized Wigner function with a realistic interaction is expected to display a similar behavior.

\section{Summary and outlook}

In this paper, we calculate kaon generalized parton distributions (GPDs) and leading Fock state light-front wave functions (LFWFs) in the Nambu-Jona-Lasinio (NJL) model using proper time regularization. We obtained form factors (FFs), generalized form factors, parton distribution functions (PDFs), impact parameter dependent PDFs, parton distribution amplitudes (PDAs) and transverse momentum dependent parton distributions (TMDs) from GPDs and LFWFs, then comparing these distributions from these two different methods, and checking their properties. 

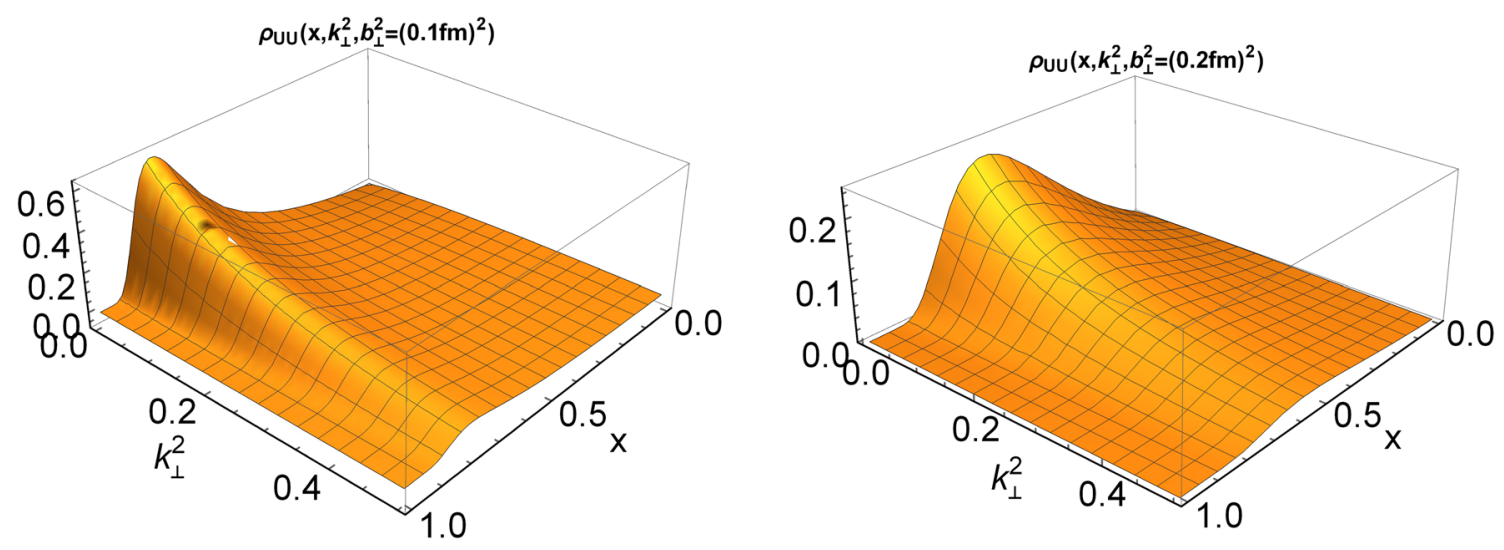

Fig. 13 The unpolarized Wigner distribution $\rho_{U U}$ at $\left|\boldsymbol{b}_{\perp}\right|=0.1 \mathrm{fm}$, and $\left|\boldsymbol{b}_{\perp}\right|=0.2 \mathrm{fm}$. The $\delta^{2}\left(\boldsymbol{b}_{\perp}\right)$ component - first line of Eq. (88) - is suppressed in the image

For the vector GPD, we find that the $u$ quark mass distribution $\theta_{2}^{u}$ and pressure distribution $\theta_{1}^{u}$ form factors are harder than its electromagnetic form factor $F_{K}^{u}$. We also study the mean-squared impact parameter for the quarks in the kaon: $\left\langle\boldsymbol{b}_{\perp}^{2}\right\rangle_{K}^{u}=0.149 \mathrm{fm}^{2},\left\langle\boldsymbol{b}_{\perp}^{2}\right\rangle_{K}^{s}=0.088 \mathrm{fm}^{2}$, which means in the kaon the $s$ quark is closer to the center of transverse momentum than the $u$ quark. For the light-cone energy radius of quarks in kaon we have $r_{E, L C}^{u, K}=0.187 \mathrm{fm}, r_{E, L C}^{s, K}=0.167$ $\mathrm{fm}$. For the light-cone charge radius of the quarks in the kaon $r_{c, L C}^{u, K}=0.390 \mathrm{fm}, r_{c, L C}^{s, K}=0.296 \mathrm{fm}$, from the results we see that in each case the $s$ quark has a smaller extent than the $u$ quark.

For the tensor GPD, we plot the diagrams of the tensor anomalous magnetic moment $B_{1,0}$ and the tensor generalized form factor $B_{2,0}$, we find that $B_{2,0}$ is harder than $B_{1,0}$.

We also study the light-front transverse-spin distributions $\rho_{u}^{1}\left(\boldsymbol{b}_{\perp}, \boldsymbol{s}_{\perp}\right)$ and $\rho_{u}^{2}\left(\boldsymbol{b}_{\perp}, \boldsymbol{s}_{\perp}\right)$, the diagrams show distortions for transversely polarized quarks. For a quark polarized in the light-front-transverse $+x$ direction, the transverse-spin density is no longer symmetric around $\boldsymbol{b}_{\perp}=\left(b_{x}=0, b_{y}=\right.$ $0)$; the peaks shift to the $\left(b_{x}=0, b_{y}>0\right)$ region. The average shift in the NJL model is $\left\langle b_{\perp}^{y}\right\rangle_{1}^{u}=0.116 \mathrm{fm}$ and $\left\langle b_{\perp}^{y}\right\rangle_{2}^{u}=0.083 \mathrm{fm}$.

From the kaon LFWF, we obtain the kaon decay constant, FF, TMD, PDF, PDA, impact parameter dependent PDF, and GPD in the region $x \in[\xi, 1]$. We obtain the same $u$ quark FF, PDF, impact parameter dependent PDF $u_{K}\left(x, \boldsymbol{b}_{\perp}^{2}\right)$ and mass distribution $\theta_{2}^{u}$ from LFWF as from vector GPD. Thus we get the conclusion that the two methods in the NJL model give the same picture of the kaon structure. In addition, we give the unpolarized Wigner distribution of the $u$ quark in the kaon. At the hadronic scale, the unpolarized Wigner function of the $u$ quark in kaon is sharply peaked in the vicinity of ( $\left.x=1, \boldsymbol{k}_{\perp}=\mathbf{0}, \boldsymbol{b}_{\perp}=\mathbf{0}\right)$, it becomes widened as the transverse position variable $\boldsymbol{b}_{\perp}$, which conjugates to the probing momentum transfer, increases in magnitude. A similar behavior should be expected of unpolarized Wigner distributions calculated with a realistic interaction.

In summary, the NJL model is proved to be quite good in describing the pion and kaon structure. In the future, on the one hand, we can generalize these calculations to other hadrons which have a more complex structure, such as the $\rho$ meson and $K^{*}$ meson, furthermore, protons and neutrons. On the other hand, we can repeat the results calculated herein using models with more realistic interactions; this may yield additional insights.

Acknowledgements We are grateful for constructive comments and technical assistance from Zhu-Fang Cui. Work supported by the National Natural Science Foundation of China (under Grant No. 11775118).

Data Availability Statement This manuscript has no associated data or the data will not be deposited. [Authors' comment: All data generated during this study are represented in this published article.]

Open Access This article is licensed under a Creative Commons Attribution 4.0 International License, which permits use, sharing, adaptation, distribution and reproduction in any medium or format, as long as you give appropriate credit to the original author(s) and the source, provide a link to the Creative Commons licence, and indicate if changes were made. The images or other third party material in this article are included in the article's Creative Commons licence, unless indicated otherwise in a credit line to the material. If material is not included in the article's Creative Commons licence and your intended use is not permitted by statutory regulation or exceeds the permitted use, you will need to obtain permission directly from the copyright holder. To view a copy of this licence, visit http://creativecomm ons.org/licenses/by/4.0/.

Funded by $\mathrm{SCOAP}^{3}$. 


\section{Appendix 1: useful formulas}

Here we use the gamma-functions ( $n \in \mathbb{Z}, n \geq 0$ )

$$
\begin{aligned}
\mathcal{C}_{0}(z) & =\int_{0}^{\infty} \mathrm{d} s s \int_{\tau_{u v}^{2}}^{\tau_{i r}^{2}} \mathrm{~d} \tau e^{-\tau(s+z)} \\
& =z\left[\Gamma\left(-1, z \tau_{u v}^{2}\right)-\Gamma\left(-1, z \tau_{i r}^{2}\right)\right], \\
\mathcal{C}_{n}(z) & =(-)^{n} \frac{\sigma^{n}}{n !} \frac{\mathrm{d}^{n}}{\mathrm{~d} \sigma^{n}} \mathcal{C}_{0}(\sigma), \\
\overline{\mathcal{C}}_{i}(z) & =\frac{1}{z} \mathcal{C}_{i}(z),
\end{aligned}
$$

where $\tau_{u v, i r}=1 / \Lambda_{\mathrm{UV}, \mathrm{IR}}$ are, respectively, the infrared and ultraviolet regulators described above.

The $\sigma$ functions are defined as

$$
\begin{aligned}
\sigma_{1}= & (1-x) M_{u}^{2}+x M_{s}^{2}-x(1-x) m_{K}^{2} \\
\sigma_{2}= & M_{u}^{2}-x(1-x) t \\
\sigma_{3}= & \frac{1-x}{1+\xi} M_{u}^{2}+\frac{x+\xi}{1+\xi} M_{s}^{2}-\frac{x+\xi}{1+\xi} \frac{1-x}{1+\xi} m_{K}^{2} \\
\sigma_{4}= & \frac{1-x}{1-\xi} M_{u}^{2}+\frac{x-\xi}{1-\xi} M_{s}^{2}-\frac{x-\xi}{1-\xi} \frac{1-x}{1-\xi} m_{K}^{2} \\
\sigma_{5}= & M_{u}^{2}-\frac{1}{4}\left(1+\frac{x}{\xi}\right)\left(1-\frac{x}{\xi}\right) t \\
\sigma_{6}= & (1-\alpha) M_{u}^{2}+\alpha M_{s}^{2}-\alpha(1-\alpha) m_{K}^{2} \\
& -\left(\frac{\xi+x}{2 \xi}-\alpha \frac{1+\xi}{2 \xi}\right)\left(\frac{\xi-x}{2 \xi}+\alpha \frac{1-\xi}{2 \xi}\right) t \\
\sigma_{7}= & (x+y)(x+y-1) m_{K}^{2}-x y t \\
& +(x+y) M_{u}^{2}+(1-x-y) M_{s}^{2} \\
\sigma_{8}= & (1-x) M_{u}^{2}+x M_{s}^{2}+(1-\alpha-x) \alpha \boldsymbol{q}_{\perp}^{2} \\
& -x(1-x) m_{K}^{2}, \\
\sigma_{9}= & z(1-z)(1-x)^{2} \boldsymbol{q}_{\perp}^{2}+x(x-1) m_{K}^{2} \\
& +(1-x) M_{u}^{2}+x M_{s}^{2} \\
\sigma_{10}= & -z(1-z) t \frac{(1-x)^{2}}{(\xi+1)^{2}(1-\xi)^{2}} \\
& +(1-z)\left(\frac{1-x}{1+\xi} M_{u}^{2}+\frac{x+\xi}{1+\xi} M_{s}^{2}+\frac{x-1}{1+\xi} \frac{x+\xi}{1+\xi} m_{K}^{2}\right) \\
& +z\left(\frac{1-x}{1-\xi} M_{u}^{2}+\frac{x-\xi}{1-\xi} M_{s}^{2}+\frac{x-1}{1-\xi} \frac{x-\xi}{1-\xi} m_{K}^{2}\right) \\
\sigma_{11}= & \boldsymbol{k}_{\perp}^{2}+m_{K}^{2}(x-1) x+(1-x) M_{u}^{2}+x M_{s}^{2} \\
\sigma_{12}= & \boldsymbol{k}_{\perp}^{2}+z(1-z) \boldsymbol{q}_{\perp}^{2}(1-x)^{2} \\
& +m_{K}^{2}(x-1) x+(1-x) M_{u}^{2}+x M_{s}^{2}
\end{aligned}
$$

\section{References}

1. D. Muller, D. Robaschik, B. Geyer, F.M. Dittes, J. Horejsi, Fortsch. Phys. 42, 101 (1994). arXiv:hep-ph/9812448

2. X.-D. Ji, Phys. Rev. D 55, 7114 (1997a). arXiv:hep-ph/9609381
3. A.V. Radyushkin, Phys. Rev. D 56, 5524 (1997). arXiv:hep-ph/9704207

4. X.-D. Ji, J. Phys. G 24, 1181 (1998). arXiv:hep-ph/9807358

5. L. Theussl, S. Noguera, V. Vento, Eur. Phys. J. A 20, 483 (2004). arXiv:nucl-th/0211036

6. M. Diehl, Phys. Rep. 388, 41 (2003). arXiv:hep-ph/0307382

7. J.-L. Zhang, Z.-F. Cui, J. Ping, C.D. Roberts, Eur. Phys. J. C 81, 6 (2021a). arXiv:2009.11384 [hep-ph]

8. J.-L. Zhang, K. Raya, L. Chang, Z.-F. Cui, J.M. Morgado, C.D. Roberts, J. Rodríguez-Quintero, Phys. Lett. B 815, 136158 (2021). arXiv:2101.12286 [hep-ph]

9. J.-L. Zhang, M.-Y. Lai, H.-S. Zong, J.-L. Ping, Nucl. Phys. B 966, 115387 (2021)

10. A.V. Radyushkin, Phys. Lett. B 380, 417 (1996). arXiv:hep-ph/9604317

11. J.C. Collins, A. Freund, Phys. Rev. D 59, 074009 (1999). arXiv:hep-ph/9801262

12. A.V. Belitsky, D. Mueller, A. Kirchner, Nucl. Phys. B 629, 323 (2002). arXiv:hep-ph/0112108

13. D. Müller, T. Lautenschlager, K. Passek-Kumericki, A. Schaefer, Nucl. Phys. B 884, 438 (2014). arXiv:1310.5394 [hep-ph]

14. L. Favart, M. Guidal, T. Horn, P. Kroll, Eur. Phys. J. A 52, 158 (2016). arXiv:1511.04535 [hep-ph]

15. M. Defurne et al. (Jefferson Lab Hall A), Phys. Rev. C 92, 055202 (2015). arXiv:1504.05453 [nucl-ex]

16. H. Jo et al. (CLAS), Phys. Rev. Lett. 115, 212003 (2015). arXiv: 1504.02009 [hep-ex]

17. D.W. Duke, J.F. Owens, Phys. Rev. D 30, 49 (1984)

18. M. Hirai, S. Kumano, T.-H. Nagai, Phys. Rev. C 70, 044905 (2004)

19. S. Dulat, T.-J. Hou, J. Gao, M. Guzzi, J. Huston, P. Nadolsky, J. Pumplin, C. Schmidt, D. Stump, C.-P. Yuan, Phys. Rev. D 93, 033006 (2016)

20. K. Wijesooriya, P.E. Reimer, R.J. Holt, Phys. Rev. C 72, 065203 (2005). arXiv:nucl-ex/0509012 [nucl-ex]

21. K.J. Eskola, H. Paukkunen, C.A. Salgado, JHEP 04, 065 (2009). arXiv:0902.4154 [hep-ph]

22. T. Nguyen, A. Bashir, C.D. Roberts, P.C. Tandy, Phys. Rev. C 83, 062201 (2011). arXiv:1102.2448 [nucl-th]

23. R.J. Holt, C.D. Roberts, Rev. Mod. Phys. 82, 2991 (2010). arXiv:1002.4666 [nucl-th]

24. P.C. Barry, N. Sato, W. Melnitchouk, C.-R. Ji, Phys. Rev. Lett. 121, 152001 (2018). arXiv:1804.01965 [hep-ph]

25. Z.-F. Cui, M. Ding, F. Gao, K. Raya, D. Binosi, L. Chang, C.D. Roberts, J. Rodríguez-Quintero, S.M. Schmidt, Eur. Phys. J. A 57, 5 (2021). arXiv:2006.14075 [hep-ph]

26. W. Broniowski, E. Ruiz Arriola, Phys. Lett. B 135803 (2020). arXiv:2006.03832 [hep-ph]

27. G. PeterLepage, S.J. Brodsky, Phys. Lett. B 87, 359 (1979)

28. A.V. Efremov, A.V. Radyushkin, Phys. Lett. B 94, 245 (1980)

29. G.P. Lepage, S.J. Brodsky, Phys. Rev. D 22, 2157 (1980)

30. J. Rodríguez-Quintero, D. Binosi, C. Chen, Y. Lu, C.D. Roberts, S. Segovia, EPJ Web Conf. 241, 02009 (2020). arXiv:1909.13793 [nucl-th]

31. I.C. Cloët, W. Bentz, A.W. Thomas, Phys. Rev. C 90, 045202 (2014). arXiv:1405.5542 [nucl-th]

32. Z.-F. Cui, C. Chen, D. Binosi, F. de Soto, C.D. Roberts, J. Rodríguez-Quintero, S.M. Schmidt, J. Segovia, Phys. Rev. D 102, 014043 (2020). arXiv:2003.11655 [hep-ph]

33. X.-D. Ji, Phys. Rev. Lett. 78, 610 (1997). arXiv:hep-ph/9603249

34. M.V. Polyakov, Phys. Lett. B 555, 57 (2003). arXiv:hep-ph/0210165

35. M.V. Polyakov, P. Schweitzer, Int. J. Mod. Phys. A 33, 1830025 (2018). arXiv:1805.06596 [hep-ph]

36. S.J. Brodsky, Y. Frishman, G.P. Lepage, C.T. Sachrajda, Phys. Lett. B 91, 239 (1980)

37. V. Chernyak, A. Zhitnitsky, Phys. Rep. 112, 173 (1984) 
38. S.J. Brodsky, G. Lepage, Adv. Ser. Direct. High Energy Phys. 5, 93 (1989)

39. S.J. Brodsky, G.F. de Teramond, Phys. Rev. Lett. 96, 201601 (2006). arXiv:hep-ph/0602252

40. G.A. Miller, B.C. Tiburzi, Phys. Rev. C 81, 035201 (2010). arXiv:0911.3691 [nucl-th]

41. S.J. Brodsky, B. Pasquini, B.-W. Xiao, F. Yuan, Phys. Lett. B 687, 327 (2010). arXiv:1001.1163 [hep-ph]

42. S.S. Chabysheva, J.R. Hiller, Phys. Rev. E 90, 063310 (2014). arXiv: 1409.6333 [hep-ph]

43. G. Beuf, Phys. Rev. D 94, 054016 (2016). arXiv:1606.00777 [hep$\mathrm{ph}]$

44. M.V. Polyakov, Nucl. Phys. B 555, 231 (1999). arXiv:hep-ph/9809483

45. M.A. Donnellan, J. Flynn, A. Juttner, C.T. Sachrajda, D. Antonio, P.A. Boyle, C. Maynard, B. Pendleton, R. Tweedie, PoS LATTICE2007, 369 (2007). arXiv:0710.0869 [hep-lat]

46. K.-C. Yang, Nucl. Phys. B 776, 187 (2007). arXiv:0705.0692 [hep-ph]

47. M. Ding, F. Gao, L. Chang, Y.-X. Liu, C.D. Roberts, Phys. Lett. B 753, 330 (2016). arXiv:1511.04943 [nucl-th]

48. C. Shi, C. Chen, L. Chang, C.D. Roberts, S.M. Schmidt, H.-S. Zong, Phys. Rev. D 92, 014035 (2015). arXiv:1504.00689 [nuclth]

49. Y. Lu, D. Binosi, M. Ding, C.D. Roberts, H.-Y. Xing, C. Xu, Eur. Phys. J. A 57, 115 (2021). arXiv:2103.03960 [hep-ph]

50. J.C. Collins, D.E. Soper, G.F. Sterman, Nucl. Phys. B 250, 199 (1985)

51. D.W. Sivers, Phys. Rev. D 41, 83 (1990)

52. A. Kotzinian, Nucl. Phys. B 441, 234 (1995). arXiv:hep-ph/9412283

53. P.J. Mulders, R.D. Tangerman, Nucl. Phys. B 461, 197 (1996). [Erratum: Nucl. Phys. B 484, 538-540 (1997)]. arXiv:hep-ph/9510301

54. D. Boer, P.J. Mulders, Phys. Rev. D 57, 5780 (1998). arXiv:hep-ph/9711485

55. C.J. Bomhof, P.J. Mulders, Nucl. Phys. B 795, 409 (2008). arXiv:0709.1390 [hep-ph]

56. V. Barone, F. Bradamante, A. Martin, Prog. Part. Nucl. Phys. 65, 267 (2010). arXiv:1011.0909 [hep-ph]

57. S.M. Aybat, T.C. Rogers, Phys. Rev. D 83, 114042 (2011). arXiv:1101.5057 [hep-ph]

58. M.G. Echevarria, A. Idilbi, A. Schäfer, I. Scimemi, Eur. Phys. J. C 73, 2636 (2013). arXiv:1208.1281 [hep-ph]

59. M.G. Echevarría, A. Idilbi, I. Scimemi, Phys. Lett. B 726, 795 (2013). arXiv:1211.1947 [hep-ph]

60. F. Hautmann, H. Jung, M. Krämer, P.J. Mulders, E.R. Nocera, T.C. Rogers, A. Signori, Eur. Phys. J. C 74, 3220 (2014). arXiv:1408.3015 [hep-ph]

61. R. Angeles-Martinez, A. Bacchetta, I. Balitsky, D. Boer, M. Boglione, R. Boussarie, F. Ceccopieri, I. Cherednikov, P. Connor, M. Echevarria et al., Acta Phys. Polon. B 46, 2501 (2015)

62. M.G. Echevarria, I. Scimemi, A. Vladimirov, JHEP 09, 004 (2016). arXiv:1604.07869 [hep-ph]

63. M. Praszalowicz, A. Rostworowski, in 37th Rencontres de Moriond on QCD and Hadronic Interactions (2002). arXiv:hep-ph/0205177

64. H. Mineo, S.N. Yang, C.-Y. Cheung, W. Bentz, Phys. Rev. C 72, $025202(2005)$

65. M.K. Volkov, A.E. Radzhabov, Phys. Usp. 49, 551 (2006). arXiv:hep-ph/0508263

66. A. Courtoy, Generalized Parton Distributions of Pions. Spin Structure of Hadrons, Other thesis (2010). arXiv:1010.2974 [hep-ph]

67. A. Courtoy, S. Noguera, S. Scopetta, JHEP 12, 045 (2019). arXiv:1909.09530 [hep-ph]
68. W. Broniowski, E. Ruiz Arriola, PoS LC2019, 031 (2020). arXiv:2001.00883 [hep-ph]

69. S. Boffi, B. Pasquini, M. Traini, Nucl. Phys. B 649, 243 (2003). arXiv:hep-ph/0207340

70. S. Scopetta, V. Vento, Eur. Phys. J. A 16, 527 (2003). arXiv:hep-ph/0201265

71. B. Pasquini, M. Pincetti, S. Boffi, Phys. Rev. D 72, 094029 (2005). arXiv:hep-ph/0510376

72. A. Vega, I. Schmidt, T. Gutsche, V.E. Lyubovitskij, Few Body Syst. 52, 237 (2012a). arXiv:1109.6449 [hep-ph]

73. G.F. de Teramond, T. Liu, R.S. Sufian, H.G. Dosch, S.J. Brodsky, A. Deur (HLFHS), Phys. Rev. Lett. 120, 182001 (2018). arXiv:1801.09154 [hep-ph]

74. N. Kaur, H. Dahiya, Eur. Phys. J. A 56, 172 (2020). arXiv:1909.10146 [hep-ph]

75. C. Mondal, D. Chakrabarti, Eur. Phys. J. C 75, 261 (2015). arXiv:1501.05489 [hep-ph]

76. D. Chakrabarti, C. Mondal, Phys. Rev. D 92, 074012 (2015). arXiv: 1509.00598 [hep-ph]

77. N. Kumar, C. Mondal, N. Sharma, Eur. Phys. J. A 53, 237 (2017) arXiv:1712.02110 [hep-ph]

78. A. Vega, I. Schmidt, T. Gutsche, V.E. Lyubovitskij, Phys. Rev. D 83, 036001 (2011). arXiv:1010.2815 [hep-ph]

79. A. Vega, I. Schmidt, T. Gutsche, V.E. Lyubovitskij, Phys. Rev. D 85, 096004 (2012b). arXiv:1202.4806 [hep-ph]

80. D. Chakrabarti, C. Mondal, Phys. Rev. D 88, 073006 (2013). arXiv:1307.5128 [hep-ph]

81. W. Broniowski, E. Ruiz Arriola, K. Golec-Biernat, Phys. Rev. D 77, 034023 (2008). arXiv:0712.1012 [hep-ph]

82. W. Broniowski, A.E. Dorokhov, E. Ruiz Arriola, Few Body Syst. 52, 295 (2012). arXiv:1108.5938 [hep-ph]

83. C. Mezrag, L. Chang, H. Moutarde, C.D. Roberts, J. RodríguezQuintero, F. Sabatié, S.M. Schmidt, Phys. Lett. B 741, 190 (2015). arXiv:1411.6634 [nucl-th]

84. S. Klimt, M.F.M. Lutz, U. Vogl, W. Weise, Nucl. Phys. A 516, 429 (1990)

85. S.P. Klevansky, Rev. Mod. Phys. 64, 649 (1992)

86. P. Rehberg, S.P. Klevansky, J. Hufner, Phys. Rev. C 53, 410 (1996). arXiv:hep-ph/9506436

87. R. Marty, E. Bratkovskaya, W. Cassing, J. Aichelin, H. Berrehrah, Phys. Rev. C 88, 045204 (2013). arXiv:1305.7180 [hep-ph]

88. N. Ishii, W. Bentz, K. Yazaki, Phys. Lett. B 301, 165 (1993)

89. M.E. Carrillo-Serrano, W. Bentz, I.C. Cloët, A.W. Thomas, Phys. Lett. B 759, 178 (2016). arXiv:1603.02741 [nucl-th]

90. D. Ebert, T. Feldmann, H. Reinhardt, Phys. Lett. B 388, 154 (1996). arXiv:hep-ph/9608223

91. G. Hellstern, R. Alkofer, H. Reinhardt, Nucl. Phys. A 625, 697 (1997). arXiv:hep-ph/9706551

92. W. Bentz, A.W. Thomas, Nucl. Phys. A 696, 138 (2001). arXiv:nucl-th/0105022

93. P. Hagler, Phys. Rep. 490, 49 (2010). arXiv:0912.5483 [hep-lat]

94. A. Freese, I.C. Cloët, Phys. Rev. C 100, 015201 (2019). arXiv:1903.09222 [nucl-th]

95. C. Shi, K. Bednar, I.C. Cloët, A. Freese, Phys. Rev. D 101, 074014 (2020). arXiv:2003.03037 [hep-ph]

96. M. Burkardt, Int. J. Mod. Phys. A 18, 173 (2003). arXiv:hep-ph/0207047

97. D. Brömmel et al. (QCDSF, UKQCD), Phys. Rev. Lett. 101, 122001 (2008). arXiv:0708.2249 [hep-lat]

98. H.H. Liu, D.E. Soper, Phys. Rev. D 48, 1841 (1993)

99. T. Heinzl, Methods of quantization. Proceedings, 39. Internationale Universitatswochen fur Kern- und Teilchenphysik, IUKT 39: Schladming, Austria, February 26-March 4, 2000, Lect. Notes Phys., vol. 572, p. 55 (2001). arXiv:hep-th/0008096

100. H. Leutwyler, Phys. Lett. 48B, 45 (1974a)

101. H. Leutwyler, Nucl. Phys. B 76, 413 (1974) 
102. W. Weise, Int. Rev. Nucl. Phys. 1, 57 (1984)

103. V. Bernard, R. Brockmann, W. Weise, Nucl. Phys. A 440, 605 (1985)

104. S. Noguera, S. Scopetta, JHEP 11, 102 (2015). arXiv:1508.01061 [hep-ph]

105. S.J. Brodsky, M. Diehl, D.S. Hwang, Nucl. Phys. B 596, 99 (2001). arXiv:hep-ph/0009254

106. M. Bastiaans, Opt. Commun. 25, 26 (1978)

107. M.J. Bastiaans, J. Opt. Soc. Am. 69, 1710 (1979)

108. H. Bartelt, K.-H. Brenner, A. Lohmann, Opt. Commun. 32, 32 (1980)

109. D.T. Smithey, M. Beck, M.G. Raymer, A. Faridani, Phys. Rev. Lett. 70, 1244 (1993)

110. X.-D. Ji, Phys. Rev. Lett. 91, 062001 (2003). arXiv:hep-ph/0304037

111. A.V. Belitsky, X.-D. Ji, F. Yuan, Phys. Rev. D 69, 074014 (2004). arXiv:hep-ph/0307383

112. C. Lorce, B. Pasquini, Phys. Rev. D 84, 014015 (2011). arXiv:1106.0139 [hep-ph]
113. B. Pasquini, C. Lorcé, Few Body Syst. 55, 287 (2014)

114. A. Mukherjee, S. Nair, V.K. Ojha, Phys. Rev. D 91, 054018 (2015). arXiv:1501.03728 [hep-ph]

115. D. Chakrabarti, T. Maji, C. Mondal, A. Mukherjee, Eur. Phys. J. C 76, 409 (2016). arXiv:1601.03217 [hep-ph]

116. Z.-L. Ma, Z. Lu, Phys. Rev. D 98, 054024 (2018). arXiv:1808.00140 [hep-ph]

117. M. Ahmady, C. Mondal, R. Sandapen, J.P. Vary, X. Zhao, in 18th International Conference on Hadron Spectroscopy and Structure (2020). arXiv:2001.01690 [hep-ph] 